\title{
All-Optical Regeneration of Phase Encoded Signals
}

\author{
Authors: \\ Dr Joseph Kakande \\ Dr Radan Slavík \\ Dr Francesca Parmigiani \\ Prof Periklis Petropoulos \\ Prof David Richardson
}

\section{Affiliation:}

All authors currently based at:

Optoelectronics Research Centre, University of Southampton

Southampton, Hampshire, SO17 1BJ, United Kingdom

Summary: In this Chapter we review the need, general principles and approaches used to regenerate phase encoded signals of differing levels of coding complexity. We will describe the key underpinning technology and present the current state-of-the-art, incorporating an appropriate historic perspective throughout. The chapter finishes with a discussion of emerging research trends and broader future perspectives. 


\section{Phase Sensitive Optical Regeneration}

\section{Introduction}

The development of the erbium-doped fibre amplifier (EDFA) revolutionized optical communications, eliminating fibre propagation loss as the primary limiting factor to transmission capacity, and enabling mass access to data transfer over inter-continental distances. By adopting wavelength division multiplexing (WDM) of high baud-rate (on-off keyed, OOK) channels, data signalling rates approaching 1Tbit/s down a single fibre were commonly deployed commercially for long haul and high capacity backbone communications by the late nineties, and the lay perception of the optical fibre was as a channel of effectively limitless bandwidth. However, due to the continued emergence of bandwidth intensive applications and services, e.g. social networking and more recently high definition video streaming, data transmission demands have continued to grow exponentially and there is an ever increasing appreciation that fibre bandwidth is a far from infinite resource: fibre bandwidth will need to be used more efficiently in future communication systems if available capacity is to stay ahead of demand for very much longer. Indeed there are fears in terms of crunches in both capacity and energy requirements ahead without further radical innovation at the physical layer. To this end the use of higher signalling rates, coupled with use of higher spectral efficiency modulation formats, has become a key research challenge.

The ability to signal in phase as well as amplitude has long been exploited in radio telecommunications to boost spectral efficiency. In fibre communications, formats such as differential phase shift keying (DPSK) and differential quadrature phase shifted keying (DQPSK) were rapidly shown to offer significant benefits over amplitude only formats, such as OOK. DPSK for example offers considerable advantages in terms of resilience to transmission impairments such as dispersion and nonlinearity, as well as an inherent $3 \mathrm{~dB}$ improvement in receiver sensitivity over OOK if balanced detection is utilised [1], and combined with sophisticated error correction techniques further improvements in system margin and/or reach are enabled. More recently, a resurgence in the use of coherent detection has allowed for an increase in spectral efficiency by the adoption of higher order modulation formats, such as coherent quadrature phase shift keying (QPSK) [2], as well as in reach due to the ability to digitally compensate, in real-time, for both linear and nonlinear transmission impairments [3]. Polarization multiplexed QPSK (PM-QPSK) has recently become the standard for long haul $100 \mathrm{Gbit} / \mathrm{s}$ optical transmission and in the laboratory far more exotic modulation formats, such as 16-QAM are routinely studied with heroic attempts being made to work with much higher complexity variants e.g. 256-QAM and above, which places great demands on system optical signal to noise ratio (OSNR) and on the underpinning component and sub-system technologies.

The reach of fibre links is ultimately limited by noise, generated either linearly due to quantum noise resulting from loss and quantum limited amplification, as well as nonlinearly due to interactions between the propagating signal and other optical waves in the same fibre [4]. Typically, amplifiers are required every $60-120 \mathrm{~km}$, compensating for around $12-24 \mathrm{~dB}$ worth of losses, and it is possible to minimise noise pickup in links by using low NF lumped amplifiers and pre-compensating for the loss by amplifying before the lossy element. Additional distributed Raman amplification is also often deployed to enhance system performance. While optical amplification can be used to substantially offset the detrimental impact of loss, the presence of optical nonlinearity means that beyond a certain peak power level, amplification degrades, rather than enhances the received signal quality. The primary nonlinear mechanism for signal quality degradation is cross phase modulation (XPM) between channels and amplified spontaneous emission (ASE), which gives rise to non-deterministic 
phase noise. For further details the interested reader is referred to reference [4] for a comprehensive review of nonlinear physical noise generation mechanisms and their impact within optical fibre communication systems.

The solution to such noise pickup is to periodically regenerate the signal, restoring it back to its original quality. Typically, commercial regenerators utilise a process referred to as Optical-ElectricalOptical (OEO) conversion. This involves the noisy optical signal (i) being detected using a photoreceiver thereby converting it into an analog electrical signal, (ii) re-digitising the analog signal thereby removing the noise, and (iii) re-modulating this digital stream onto a new non-degraded optical carrier. Digital error correction can also be used on the electrical signal, enhancing the performance of the system significantly. Whilst such an approach potentially allows transmission over essentially unlimited distances, the clear drawback is that the OEO conversion and associated electronic processing substantially restricts the speed and latency of the system, moreover there are associated power consumption/heat dissipation issues which are ultimately limiting as the bit rate is increased. The ideal solution to these problems would then be to perform the regeneration function all optically.

Over the years there has been substantial work on the problem of all-optical regeneration with many interesting and impressive results achieved in the laboratory [5]. However, given that most research over the past 30 years has concerned the regeneration of simple intensity modulated signals the majority of this work is unfortunately now largely redundant given the clear and necessary migration to more complex phase based optical signalling formats. The challenge in the field of optical regeneration has shifted and is now to develop regeneration techniques that can operate on complex, phase based modulation format signals. Work in this area only really started some 5-6 years ago and the purpose of this Chapter is to review the impressive progress made to date.

All optical phase regeneration can be approached in a number of distinct ways. The most obvious way, perhaps, is to try and exploit all the work done to date on amplitude-encoded signals by providing some means to convert an incident phase-encoded signal to an amplitude-encoded signal, to regenerate that all optically, before converting back to the original (regenerated) phase-encoded format. To an extent this can be viewed as the phase-to-amplitude converter analogue of the OEO regenerator described previously. A more attractive approach however is to exploit optical processes which are inherently sensitive to optical phase - of which the Optical Parametric Amplifier (OPA), and more specifically the Fibre OPA (FOPA) is the archetypal example [6]. FOPAs have a number of interesting modes of operation and performance features that make them especially attractive for manipulating advanced modulation format signals.

In conventional Phase Insensitive (PI-) mode, in which there is no well-defined phase relationship between the waves involved (Signal, Idler and Pump(s)), FOPAs can be used to limit the intensity of signals via a gain saturation mechanism [7]. This has been used to regenerate the intensity of DPSK signals [8], reducing the nonlinear pickup of noise in transmission spans following the regenerator, with demonstrations showing reach enhancement [9]. The extinction ratio of OOK signals can also be increased using a higher-order four wave mixing (FWM) effect in FOPAs, allowing for another optical regeneration mechanism [10].

In Phase Sensitive (PS) mode, two pump symmetric (degenerate) FOPAs can directly eliminate phase noise from DPSK signals due to their $\pi$-periodic step phase characteristics. When saturated, they can also use the same amplitude limiting function described earlier to eliminate amplitude noise [11]. This 
combination of effects can be used to perform simultaneous phase and amplitude regeneration of DPSK signals $[11,12]$. As we shall describe later this phase regeneration capability can be used to extend the reach of DPSK-bearing fibre links. This configuration however is limited to two-level PSK signals, and not the higher order modulation formats such as QPSK that are superseding DPSK. In order to work on higher modulation formats it is necessary to cascade multiple parametric effects to build up the necessary device transfer functions [13].

In this chapter we review the general principles and approaches used to regenerate phase encoded signals of differing levels of coding complexity. We will describe the key underpinning technology and present the current state-of-the-art. This chapter is structured as follows: We first review the different approaches and nonlinear processes that can be used to perform the regeneration of phase encoded signals. Our primary focus is on parametric effects, which as we have explained previously can operate directly on the optical phase. We then proceed to review progress on regenerating the simplest of phase modulation formats, namely DPSK/BPSK and for which the greatest progress has been made to date. We then move on to discuss progress in regenerating more complex modulation format signals: in particular (D)QPSK and other M-PSK signals. Next we review the choice of nonlinear components available to construct phase regenerators. It is not our intention to provide a rigorous and complete overview of all options in this section but rather to describe the various merits and issues associated with each component type and material system. Finally, we review the prospects for regenerating even more complex signals including QAM and mixed phase-amplitude coding variants. We end with a brief summary and conclusions.

\section{Approaches to Regeneration of Phase Encoded Signals}

For the purpose of this review we classify a PSK regenerator in the broadest of terms as a device that actively reduces the accumulated phase noise between an optical PSK transmitter and receiver. However, before we begin, it is worth noting the whole host of other (passive) ways to achieve the same goal, such as installing lower noise amplifiers [14], using transmission fibre with reduced loss and/or nonlinearity [15], and optimising the link dispersion map [16]. The regenerators discussed in this chapter post-compensate either for accumulated phase noise, or for amplitude noise that could seed phase noise via nonlinear mechanisms during further propagation [4]. Given the variety of physical mechanisms that can create phase noise within an optical fibre link, multiple approaches have been proposed to actively perform regeneration, each seeking to balance the complexity of implementation with the required level of performance.

It is useful to divide the schemes for optical phase regeneration into two broad categories, direct and indirect. Direct regenerators operate on the premise that a signal has already been degraded in phase, and therefore signal quality can only be improved by suppressing these phase perturbations. To perform direct phase regeneration, a method of distinguishing between the noise and signal, both of which occur in phase, is required. Indirect regenerators act by suppressing amplitude fluctuations which could seed phase noise further on - eliminating the need to distinguish between the signal, which occupies phase space, and noise, located in the amplitude domain. Note that the process of amplitude noise suppression must not create significant added phase noise, to make it worthwhile.

\subsection{Indirect PSK regeneration}

Indirect phase regeneration is often referred to as phase preserving amplitude regeneration, which is rather apt as it describes the actual mechanism involved in the regenerator. The devices are also 
referred to in the literature as limiters, as they limit signal power to a narrow range. It is suitable in systems where nonlinear phase noise is the dominant limiting factor in receiver bit error rate (BER) [17]. The historical use of OOK in optical links means that all-optical amplitude regeneration has been extensively investigated in the past, predominantly using nonlinear (power-dependent) optical effects such as nonlinear refraction and parametric gain saturation. Many of the schemes, however, cannot be used for indirect phase regeneration as they intrinsically transform the amplitude noise into the phase (or frequency) domain. It is worth clarifying that every all-optical amplitude regenerator will add some phase noise, although some do so to a lesser extent, and therefore more tolerably, than others. We highlight some key examples here.

\subsubsection{Nonlinear Loop Mirror}

\section{FIGURE 1 GOES HERE}

Nonlinear optical loop mirrors (NOLMs) transfer the optical signal from an input to an output port with a power dependent transfer function, mediated by the interference between two powerimbalanced counter-propagating portions of the signal within a loop comprising a Kerr optical material [18]. The nonlinear transfer function is obtained due to the different amounts of phase modulation induced on the signal via the Kerr effect. A plateau in the output signal power can then be obtained whilst providing minimum phase distortion [19] by optimizing the device configuration and by defining the input signal dynamic range. Stephan et al. [20] demonstrated one such device, referred to as a nonlinear amplifying loop mirror (NALM) due to the insertion of a gain element (EDFA) inside the loop. By installing the NALM inside a re-circulating loop with $100 \mathrm{~km}$ transmission per span, they observed significant improvement in launch power margin, Figure 1, hence superior link BER [20].

\subsubsection{Four Wave Mixing based limiting}

\section{FIGURE 2 GOES HERE}

FWM is another process that can generate a plateau in its amplitude transfer function without significantly distorting the phase [6, 21]. In [21], Matsumoto demonstrates a FWM limiter (Figure 2a) installed either before, or within a $5 \mathrm{span}, 200 \mathrm{~km}$ recirculating loop (40 km per span). The setup used for characterizing the regenerative properties of the limiter for $10 \mathrm{GHz}$ return-to-zero DPSK data signals is reproduced in Figure $2 \mathrm{~b}$. Note that the signal OSNR after the transmitter (before limiting) was deliberately degraded to around $21 \mathrm{~dB}$, to ensure that there was enough amplitude noise on the signal for the limiting to provide a significant improvement. The results obtained (shown in Figure 2d) clearly show the improvement obtained with periodic regeneration [21]. Other results presented in [21] confirmed that the improvement was due to the reduction in phase noise build up along the transmission line.

\subsubsection{Optical Injection Locking}

The phase and amplitude transfer characteristics of injection-locked semiconductor lasers have also been proposed and demonstrated as a means to perform limiting of PSK signals [22, 23]. By carefully choosing the power of the master (input signal), the slave laser can follow all phase changes on the master up to a limit in frequency, while holding the output amplitude constant. Being optoelectronic 
in nature the bandwidth is currently limited to just a few tens of GHz. The technique has the benefit of being very simple in terms of implementation, and lends itself well to integration.

\subsubsection{Saturable Absorption}

A novel multiple-quantum-well (QW) semiconductor saturable absorber structure has also been demonstrated to be capable of performing amplitude limiting at over $40 \mathrm{Gbit} / \mathrm{s}$ for DPSK input signals [24]. Unlike conventional saturable absorbers, the reflectance of the device decreases when the incident optical power increases, allowing for a power limiting function when operated in reflection. Le et. al. [24] demonstrated the device installed prior to a $100 \mathrm{~km}$ transmission span, obtaining an extra $2 \mathrm{~dB}$ margin in fibre launch power. The demonstration has also been recently extended to DQPSK [25]. The device is fully passive, eliminating the need for active cooling or electrical biasing, although a practical implementation may require signal pre-amplification.

\subsection{Direct phase regeneration}

The experiments referred to in Section 2.1 all utilised signals degraded with a lot of amplitude noise, but low levels of phase noise, prior to a long transmission. Under those circumstances the limiter does reduce the amplitude-to-phase noise conversion process. However, in cases where phase noise has already been acquired, a direct phase regenerator is required to suppress the noise. We discuss some of the alternatives here.

\subsubsection{Format conversion based regeneration}

As mentioned earlier, various schemes for amplitude regeneration have been investigated in the past, offering trade-offs between regeneration performance and system complexity. Format conversion based regenerators leverage these schemes, and rely on optical techniques to convert the optical PSK signal to one or more OOK signals. The OOK signals can then be regenerated in amplitude, without the requirement to be phase-preserving, after which the now 'clean' amplitude information is transferred back on to the phase of a noise-free optical carrier.

Converting the PSK signal into OOK is done using interference, either differentially or fully coherently. Doing so differentially, in a delay line interferometer (DLI) is the simplest approach, although the logical states of the input signal get changed in the process, albeit in a deterministic fashion, and therefore care must be taken in decoding the signal at the receiver [6]. The conversion can also be done by interfering the signal with a phase-locked local oscillator, eliminating the logicalteration problem in the differential case, although performing the phase-locking represents a significant technical challenge in itself.

\section{FIGURE 3 GOES HERE}

In [21], a delay line interferometer (DLI) was used to convert a DPSK signal into an amplitude encoded signal before the regenerative step and format re-conversion. A self-phase modulation (SPM) based fibre device was used to regenerate the OOK signal which was followed by an all-optical OOKPSK conversion device using cross-phase modulation (XPM). The general scheme is shown in Figure 3 . The regenerative capabilities of one such device are presented in $[6,21]$. It was demonstrated that 
the regenerator could, at least up to a certain level of nonlinear noise, significantly reduce the receiver power penalty, although for higher levels of nonlinear phase noise some error flooring was observed.

\subsubsection{Phase sensitive amplification}

Phase sensitive amplification (PSA) has been widely recognized as an effective way to regenerate phase-encoded signals thanks to its phase squeezing capabilities [26, 27]. It has also been shown to regenerate the amplitude of the signal when operated in the saturated regime [27]. While a format conversion based device requires knowledge of the signal symbol rate, as well as recovery of the signal clock so as to perform amplitude regeneration, PSAs operate directly on the input signal field, and can be incorporated in-line using continuous wave pumps. They also allow the possibility of low noise amplification, although when used as regenerators this feature is not required. These benefits have spurred significant research efforts into PSAs in recent times, and we summarise key results in this field in the following sections.

\section{PSA based phase regeneration}

Phase sensitive optical amplifiers exhibit gain characteristics that depend on the phase of the input optical signal relative to some local optical references. The theory of PSAs is more than five decades old, and interest in them first stemmed from a semi-classical realisation that knowledge of signal phase allows signal measurements to be made more precisely than the Uncertainty Principle dictates [28], and later from a more in-depth analysis by Caves [29] that showed how this could be used for noiseless amplification. Research efforts into PSA increased significantly due to the new appreciation of their ability to squeeze the noise added to any input light below the quantum limit in one of the two phase quadratures; an excellent review by Slusher and Yurke [30] highlights the diverse applications of squeezed light in communications and sensing. Second order nonlinearity in bulk crystals was used to demonstrate degenerate phase sensitive amplification, both for squeezing [30] and amplification below the $3 \mathrm{~dB}$ quantum noise limit [31].

Practical PSAs deployed within optical networks would allow significant benefits - increased receiver OSNR allowing more spectrally efficient communications, increased amplifier spans and interesting photon correlation characteristics of possible interest for quantum communications. The ability to provide different gains to the two phase quadratures can also be used for all-optical signal processing, including phase regeneration and signal sampling/characterisation. Performing PSA using third order nonlinearity in optical fibres rather than in second order crystals was a first step towards practical network applications due to the increased robustness, improved power efficiency and ease of system integration. Marhic et al. successfully demonstrated the first degenerate PSA by 1991 [32] but progress in the field was quite limited up until the demonstration of amplification with $1.8 \mathrm{~dB}$ NF by Imajuku et. al. in 1999 [33]. Since then, PSAs have been demonstrated operating over wide bandwidths $[34,35]$ as well as with a record low noise figure [36].

PSAs principally operate by amplifying one of the input signal's quadratures and de-amplifying the other [32]. This is very convenient for BPSK regeneration because signal information is distinguishable from the noise because they can be made to occupy orthogonal quadratures $[11,26]$. While PSA can be obtained in various media (as discussed later on in the chapter), we focus on FOPA 
implementations here as FOPAs devices comprise the majority of PSA-based phase regenerators in the literature. We discuss the theory behind phase sensitive (PS) -FOPAs in this section and highlight key experimental demonstrations of regeneration.

\subsection{Binary phase quantization theory}

The direct phase regeneration of BPSK signals requires a device capable of amplifying a signal's inphase component, while de-amplifying the quadrature component. One such device is the PSA. PSFOPAs can be achieved via interferometric (Fig. 4, left) or non-interferometric means (Fig. 4, centre). As experimentally demonstrated in [37], conventional PSAs squeeze the input phase to one of two $\pi-$ separated phase levels, accompanied by a sinusoidal phase-to-amplitude transfer characteristic (Fig. 4, right). The theory of both interferometric and non-interferometric PSAs is discussed as follows.

\section{FIGURE 4 GOES HERE}

\subsubsection{Interferometric PSA}

BPSK regeneration was in fact first proposed and demonstrated using degenerate PSA in a Sagnac loop [26]. Figure 4 (left) shows the setup for a typical interferometric PS-FOPA, based around a Sagnac interferometer. A $3 \mathrm{~dB}$ splitter is used to combine a strong pump with a signal, both of which are phase locked at the same frequency. The outputs of the splitter are then coupled into the opposite ends of a nonlinear fibre, allowing the combined beam from each splitter port to travel separately from that emerging from the other splitter port. During propagation in the fibre, the two beams experience a nonlinear phase, after which they are recombined in the splitter. An amplified version of the signal can be seen to emerge from the same splitter input port as the original signal and can be retrieved using a circulator as shown, while the pump exits the loop at the other splitter port. A modification to this setup would be to send the two combined outputs through separate fibres and have a second $3 \mathrm{~dB}$ splitter to separate the signal and pump, effectively creating a nonlinear MachZehnder interferometer, but this requires two length-matched nonlinear fibres and is susceptible to acoustic and thermally induced phase perturbations and hence is more unstable.

The gain expression for this device can be found in a straightforward fashion. Assuming $P_{s}$ and $P_{p}$ to be the input signal and pump powers respectively, $\gamma$ to be the nonlinear coefficient of the fibre, $L$ to be the effective length of the fibre and $\phi$ to be the phase difference between signal and pump at the splitter, then the output signal power $P_{S}$ can be found as $[32,38]$

$$
P_{S}(\phi)=P_{S} \cos ^{2}\left(\Phi_{N L} \cos (\phi)\right)+P_{p} \sin ^{2}\left(\Phi_{N L} \cos (\phi)\right)-\sqrt{\left(P_{p} P_{S}\right)} \sin (\phi) \sin \left(2 \Phi_{N L} \cos (\phi)\right),
$$

where

$$
\Phi_{N L}=\gamma L \sqrt{P_{s} P_{p}} .
$$

Note that Eqn. 1.1 assumes that the fibre dispersion is negligible (a valid assumption for narrowband signals) and the losses are small. If $P_{p} \square P_{s}$ and $\Phi_{N L} \square 1$, then the small angle approximation can be applied to Equation 1.1, giving

$$
P_{S}(\phi)=P_{S}\left(1-\frac{\Phi_{N L}^{2}}{2} \cos ^{2}(\phi)\right)+P_{p} \Phi_{N L}^{2} \cos ^{2}(\phi)-2 \Phi_{N L} \sqrt{\left(P_{p} P_{s}\right)} \sin (\phi) \cos (\phi) .
$$


It can be seen from Eqn. 1.3 that the signal gain will vary with phase $\phi$ with $\pi$ periodicity. The solutions for the maximum and minimum gain, $G_{1}$ and $G_{2}$ respectively are [38]:

where

$$
\begin{aligned}
& G_{1}=1+2 \Phi^{2}+2 \Phi \sqrt{1+\Phi^{2}} \\
& G_{2}=1+2 \Phi^{2}-2 \Phi \sqrt{1+\Phi^{2}}
\end{aligned}
$$

$$
\Phi=\frac{\gamma L P_{p}}{2}
$$

Because $G_{1} \cdot G_{2}=1$, it can be calculated (not shown here) that the noise figure for such an amplifier would be $0 \mathrm{~dB}$, for a more detailed quantum-mechanical analysis see Shirasaki et al [39]. Note that the maximum gain varies with $\left(\gamma L P_{p}\right)^{2}$; this will be referred to shortly while comparing interferometric and non-interferometric PSAs.

Croussore et al. [26] reported the first experimental demonstrations of DPSK regeneration using an interferometric PSA in the setup shown in Figure 5. A DPSK signal was degraded in phase and amplitude using a single frequency tone imparted onto inline electro-optic modulators, and the ability of the device to suppress both forms of noise noted, Figure 5 (right). For amplitude regeneration, a plateau in the amplitude transfer function of the loop mirror was used, similar to that described in section 2.1 .

\section{FIGURE 5 GOES HERE}

\subsubsection{Non-interferometric PSA}

Fibre based BPSK regeneration in optical fibre can also be obtained using the non- interferometric degenerate PSA, Figure 4 (centre, b). For the dual pump (degenerate) PS-FOPA, the gain function can be written as $[35,40]$

$$
G_{s}(z)=\cosh (g z)+\frac{i}{g} \frac{\kappa}{2} \sinh (g z)+\frac{1}{g} r \exp \left(-i\left(\phi_{\text {rel }}-\frac{\pi}{2}\right)\right) \sinh (g z),(1.7)
$$

where $r=2 \gamma \sqrt{P_{1} P_{2}}$ and the parametric gain coefficient $g=\sqrt{r^{2}-\frac{\kappa^{2}}{2}}$. The phase mismatch $\kappa$ is a measure of how the pump(s) and signal(s) walk-off in phase relative to each other, and can be minimised by compensating for chromatic dispersion with nonlinear refraction [40, 41]. In a well phase matched parametric amplifier, $\kappa \approx 0$, therefore Eqn 1.7 reduces to

$$
G_{s}(z) \approx \cosh (g z)+\exp \left(-i\left(\phi_{r e l}-\frac{\pi}{2}\right)\right) \sinh (g z) .
$$

By setting the sum of the pumps' phases as the phase reference, then

$$
G_{s}(z) \approx \cosh (g z)+\exp \left(-i\left(2 \phi_{s}-\frac{\pi}{2}\right)\right) \sinh (g z)
$$

And by using the identity $\cosh ^{2}-\sinh ^{2}=1$, Eqn. 1.9 further reduces to 


$$
G_{s}(z) \approx \cosh (g z)\left[1+\exp \left(-i\left(2 \phi_{s}-\frac{\pi}{2}\right)\right) \tanh (g z)\right]
$$

Assuming an input analytical signal $\exp \left(i \phi_{s}\right)$, then the output of the PSA can be obtained by multiplying the analytical input with the gain function, hence

$$
\exp \left(i \phi_{s}\right) G_{s}(z) \approx \cosh (g z)\left[\exp \left(i \phi_{s}\right)+\exp \left(-i\left(\phi_{s}-\frac{\pi}{2}\right)\right) \tanh (g z)\right]
$$

Observe that $\tanh (g z)$ is bounded within the range \pm 1 , and that $\cosh ^{2}(g z)$ gives the peak power gain.

The first experimental demonstration of such a regenerator was reported by Croussore et. al. [12]. As shown in Figure 6, two pumps were generated by applying sinusoidal modulation and sideband filtering of the same optical carrier as the signal (tapped off before the modulation). The pumps, 40 $\mathrm{GHz}$ on either side of the signal, were subsequently amplified and combined with the noisy data signal. Noise was emulated via amplitude and phase modulation at a single frequency, and the regenerator was found to cope well with both the phase and amplitude noise. For amplitude regeneration, saturation of the FWM process was exploited, similarly to FWM-based amplitude limiters. Constellation diagrams clearly showing regeneration in both the phase and amplitude are also shown in Figure 6 (right).

\section{FIGURE 6 GOES HERE}

\subsubsection{Comparison of interferometric and non-interferometric PSAs}

From the expressions for the maximum gain of the interferometric PS-FOPA, Equation 1.4, and that of the phase-matched non-interferometric non-degenerate PS-FOPA, Equation 1.10, it can be seen that in the case of the former, the gain scales quadratically with pump power as compared to exponentially in the latter. As an example, for a fibre of nonlinear coefficient $12 / \mathrm{W} / \mathrm{km}$ and effective length $200 \mathrm{~m}$ (typical values), the expected gains in interferometric and non-interferometric mode are shown in Figure 7, calculated analytically.

\section{FIGURE 7 GOES HERE}

For these parameters, achieving $20 \mathrm{~dB}$ gain would require $4 \mathrm{~W}$ of pump power in the interferometric PSA, as compared to $1 \mathrm{~W}$ in the non-interferometric. In addition, the interferometric PSA is inherently single channel, while the non-interferometric device can operate with multiple signals provided that corresponding idlers are presented at the device input. Non-interferometric PSAs are therefore more energetically efficient, and therefore the preferred choice for most recent demonstrations of BPSK regeneration.

\subsection{Saturation of PSAs for amplitude noise suppression}

The phase regeneration function in degenerate PS-FOPAs is typically accompanied by phase-toamplitude conversion [11]. As a result phase noise at the PSA input is transformed into amplitude noise at the output. Consequently, if such a PSA was used as a mid-span optical phase regenerator, the amplitude noise added within the regenerator would act to generate more phase noise in the next transmission span, reducing the overall benefit of having a phase regenerator in the first place. It therefore becomes crucial when PSA based phase regenerators are deployed mid-span to saturate the 
PS-FOPAs (by boosting the input signal power), so as to clamp the maximal signal gain, and minimise the amplitude fluctuations seen on the signal at the PSA output [11].

\subsubsection{Experimental observation of saturation in degenerate PS-FOPA}

\section{FIGURE 8 GOES HERE}

In [42], an experiment was performed to study the saturation process in a FOPA and demonstrates the amplitude noise suppression in a PS-FOPA phase regenerator. The setup is shown in Figure 8. The PSA was a dual pump degenerate device, with the pumps $200 \mathrm{GHz}$ either side of the signal in frequency. The phase of the signal wave was modulated using a $50 \mathrm{kHz}$ ramp function applied to a piezo-electric fibre stretcher, allowing the phase-to-amplitude conversion to be observed at the PSA output.

\section{FIGURE 9 GOES HERE}

The normalised signal gain curves are shown in Figure 9. At the lowest signal level assessed, a sinusoidal gain curve was obtained indicating the absence of significant gain saturation. As the signal power increased the gain curve is seen to acquire a flat-top. Because each curve was captured for a fixed signal power at the PS-FOPA input, this differs from PI saturation (in which the gain varies with input signal power) because a nonlinear dependence on input signal phase exists. In this case, every point on the curve experiences a gain shift due to the alteration of the phase matching condition by the variation in the signal/pump power proportions during propagation. As the signal and pumps propagate down the fibre in which the PS interaction occurs, at every point a nonlinear contribution to the phase matching exists as a result of SPM and XPM respectively. Because the gain is dependent on the signal phase, the absolute powers and therefore the nonlinear phase matching varies with the input phase. For strong signal levels, this phase dependent behaviour leads to a modification of the gain characteristic, and ultimately to gain saturation. These results were validated by similar observations in [43].

\section{Black-box PSA based BPSK Regeneration}

To advance the work done by Croussore et. al [12], several key issues needed to be addressed. Firstly, a field-installed PSA requires a means of phase locking the pump(s) to the input signal. A temporary work-around of this issue was employed in the demonstrations of Ref. [12] by using the same laser for both pump(s) and signal. Maintaining the phase relationship between local free-running pump(s) and the incoming BPSK signal is challenging in practice because phase-encoded signals have their carrier suppressed. Consequently, techniques such as injection locking that work for OOK carrier recovery [44] cannot be used without placing restrictions on the nature of the data pattern applied to the signal [45].

In addition to phase locking, the ability of the device to cope with real-world impairments, such as wideband noise in phase and frequency, residual chromatic and polarization mode dispersion, and slow polarization drifts, needed to be considered. All of these issues are discussed in this section.

\subsection{Phase locking of pumps and signal}


To perform optical carrier recovery from a BPSK signal, a number of options are available. Feed-back $[46,47]$ or feed-forward methods [48] both exist. Alternatively the carrier can be transmitted in a separate polarization or frequency channel to the signal [49], or the data coding may be modified to leave some residual component of the carrier in the data spectrum [45]. The feedback methods require short loop delays to achieve reasonable $(>1 \mathrm{MHz})$ bandwidths, something that is ultimately limited by the physical layout of electronic and optical devices. The electrical feed-forward schemes are generally limited to processing signals with modest baud rates (e.g., in [48], 10 Gbaud signals are processed with $>10 \mathrm{GHz}$ electronics) although an optically-assisted scheme that can use electronics slower than the data baud rate was recently reported [50]. In that method, the inherently ultrafast FWM is used to down-convert the carrier variations to the baseband $(<10 \mathrm{GHz})$ allowing subsequent electronic-based processing before transfer back into the optical domain to obtain the underlying carrier frequency.

In [51, 52], a simple method to synthesize the phase locked pumps is shown, which is described here in detail. If $\varphi_{\text {Pump } 1}, \varphi_{\text {Pump } 2}$ and $\varphi_{\text {Carrier }}$ refer to the absolute phases of the two pumps and signal carrier respectively, then 'classic' phase locking of all three waves would require the conditions

$$
\varphi_{\text {Pump } 1}=\varphi_{\text {Pump } 2} \text { and } \varphi_{\text {Carrier }}=\varphi_{\text {Pump } 2}
$$

In the case of the regenerator, the requirement is to lock the relative, rather than absolute phases, and therefore the condition is

$$
\varphi_{\text {Pump } 1}+\varphi_{\text {Pump } 2}=2 \varphi_{\text {Carrier }} .
$$

This condition is much simpler to satisfy than that in Equation 1.12. First, a free running pump (Pump 1 ) is mixed with the input signal of phase $\varphi_{\text {Signal }}$ (which is a combination of the signal carrier as well as the overlying phase modulation) to generate an idler via FWM. The nature of the FWM process means that the idler's phase is [53]

$$
\varphi_{\text {Idler }}=2 \varphi_{\text {Signal }}-\varphi_{\text {Pump } 1} \text {. }
$$

The BPSK signal comprises a carrier with a phase shift of either 0 or $\pi$ and thus

$$
2 \varphi_{\text {Signal }}=2(0 \text { or } \pi)+2 \varphi_{\text {Carrier }} \equiv 2 \varphi_{\text {Carrier }} .
$$

Substituting Equation 1.15 into 1.14 yields

$$
\varphi_{\text {Idler }}=2 \varphi_{\text {Carrier }}-\varphi_{\text {Pump } 1}
$$

which has two important consequences. First, the idler does not bear the data modulation. Secondly, the idler phase is exactly what is required for the phase of Pump 2, as follows from Equation 1.13. Direct amplification of the idler to serve as pump 2 would not provide good performance, partly due to its poor OSNR (it carries all the amplitude noise present on the input signal, and it can also be quite weak in power). Also, some of the high frequency phase noise present on the data signal is also present on the idler [50]. Optical injection locking of a semiconductor laser to the noisy idler [50] can be used to address both of these issues for the following reasons. Firstly, injection locking inherently allows regeneration of signal amplitude [22]. By reducing the injected power, the injection locking bandwidth can be reduced, meaning that any high-frequency noise present on the idler is not transferred to the injection locked laser output. Secondly, an additional benefit of injection locking is the significant amplification of the weak idler (by gains of up to $60 \mathrm{~dB}$ or more), as needed for the synthesis of Pump 2. 
The practical implementation as demonstrated in [51] is shown in Figure 10. Pump 1 was combined with the data signal and sent to a highly nonlinear fibre (HNLF) to generate a modulation-stripped idler that was then used to injection-lock a slave laser and realise pump 2.

FIGURE 10 GOES HERE

\subsection{Noise suppression capability of the black-box regenerator}

As mentioned previously, noise generated inside a real fibre network can be divided into that generated by linear processes (typically ASE from EDFAs) and non-linear processes (e.g. XPM between the signal and ASE noise). For laboratory experimentation, the former source of noise ('linear noise') can be emulated simply by directly adding ASE to the signal, and this technique was used in [51]. The latter ('nonlinear noise') being predominantly phase noise was emulated in the work described below by driving a large-bandwidth $(40 \mathrm{GHz})$ phase modulator with broadband electronic noise. This electronic noise was generated by feeding ASE into a fast photodiode (16 GHz bandwidth) and amplifying the resultant signal [51]. The set-up of the noise emulator is shown in Figure 11.

\section{FIGURE 11 GOES HERE}

\section{FIGURE 12 GOES HERE}

The full regenerator set-up is shown in Figure 12. The input was a noisy DPSK signal, the polarization of which was automatically aligned to the regenerator's principal polarization axis using a polarization tracker. For phase synchronization of the pumps, the scheme shown in Figure 10 was used. The pumps were $200 \mathrm{GHz}$ away from signal. Pump 1 was a $\mathrm{CW}$ laser emitting $17 \mathrm{dBm}$ of power. After HNLF 1, the three waves (Data signal, Pump 1, Idler) were separated in a 4-channel 200-GHz demultiplexer placed behind a circulator. A mirror provided retro-reflection in the data path as well as in the path of Pump 1, which also included a piezoelectric transducer (PZT) fibre stretcher and a variable attenuator. A Discrete Mode (DM) semiconductor laser [54], which was injection locked to the idler, was used to generate Pump 2 in the idler path.

The data signal with the two (phase-locked) pumps was then launched into the PSA which consisted of a high-power EDFA (total power of $31 \mathrm{dBm}$ ) and a 180-m sample of a high stimulated Brillouin scattering (SBS)-threshold HNLF (HNLF 2) [55, 56]. HNLF 2 had an alumino-silicate core and had a linear strain gradient applied along its length (ranging from 400 to $20 \mathrm{~g}$ ) to broaden the SBS gain bandwidth and reduce the peak gain. For optimum performance the PSA was operated in deep saturation (see Section 3.2).

The BER at the regenerator input and output was recorded at $40 \mathrm{Gbit} / \mathrm{s}$ for various levels of noise, generated as shown in Figure 11. An optically-preamplified receiver and a 1-bit delay interferometer (DLI) for data demodulation was used to detect the data. In Figure 13a, it can be seen that no power penalty with respect to the back-to-back is observed when there is no noise at the PSA input. For all levels of phase-only (Figure 13a), ASE-only (Figure 13b) and combined noise (Figure 13c), we see that there is some error flooring for the signal both with and without the regenerator. By comparing Figure 13a and Figure 13b it can be seen that the regenerator copes better with 'non-linear' (phaseonly) phase noise than with 'linear' noise (ASE), which is in line with the theoretical predictions discussed in [57]. The reader is referred to [57] for an in-depth discussion of link performance with such a regenerator inline.

\section{FIGURE 13 GOES HERE}


A further issue to consider is the effect of residual dispersion on the performance of the regenerator. Constellation diagram analysis, Figures $14 \mathrm{a}$ and $14 \mathrm{~b}$, show that the regenerator is capable of reducing the phase noise fluctuations even in the presence of some residual dispersion $(80 \mathrm{ps} / \mathrm{nm}$ in the diagrams shown) albeit at the expense of increased amplitude stability. This influences the BER as shown in Figure 14c (taken from [58]), where it can be seen that the regenerator can cope well with residual dispersion corresponding to residual dispersion accumulated in about $2 \mathrm{~km}$ of SMF-28, but gives no advantage for dispersion accumulated in an SMF-28 length of $8 \mathrm{~km}$. More discussion regarding this topic can be found in [58].

\section{FIGURE 14 GOES HERE}

Another potentially useful feature of the regenerator is its inherent capability of wavelength multicasting through cascaded FWM processes, as explained in Figure 15. As sketched in this figure, several FWM interactions may occur simultaneously: PSA, which involves the two pumps and the data, is responsible for the regeneration (Figure 15a), whereas new (secondary) pumps as well as new copies of the data signal are generated via phase insensitive FWM interactions (Figure 15b). In the saturation regime, the high input signal power gives rise to the generation of multiple FWM products which inherit the amplitude and phase properties of the initially interacting waves (Pump 1, Pump 2, and Data). Theoretical and experimental analysis showing that the generated signal copies are also regenerated is presented in [57]. This feature was used for performing simultaneous regeneration and wavelength conversion in a field trial experiment as described later. This feature is shown in the regenerator setup in Figure 12 showing two outputs from the regenerator (at the original wavelength and at a wavelength-shifted wavelength). In practice, up to 5 output copies were reported [57], Figure $15 \mathrm{c}$.

\section{FIGURE 15 GOES HERE}

\subsection{Field results with DWDM network-generated noise}

A field transmission experiment which made use of the wavelength conversion/multicasting feature of the regenerator was carried out [58]. A conceptual outline of the network and its practical implementation are shown in Figure $16 \mathrm{a}$ and $\mathrm{b}$ respectively. At the transmitter, $37 \mathrm{CW}$ semiconductor lasers were combined on a $100 \mathrm{GHz}$ dense wave division multiplexing (DWDM) ITU grid and modulated with $40 \mathrm{Gbit} / \mathrm{s}, 2^{31}-1$ pseudo-random bit sequence (PRBS) DPSK data. To de-correlate adjacent channels, the odd/even channels were split in an interleaver, $55 \mathrm{~ns}$ of relative delay was introduced, and the channels then re-combined. In order to facilitate placing the regenerator at the mid-point of the network and to operate at twice the transmission distance that was physically available, a wavelength shift was incorporated in the regeneration process, Figure 16 b. Obviously, conversion of the wavelength would not normally be required in most transmission applications, nevertheless, it serves to illustrate another useful functionality of the technology (wavelength conversion/multicasting) as previously reviewed [57, 58]. 37 channels (excluding ITU Channel 23) were sent down the dark fibre link (part of the UK JANET Aurora Network) that extends from Southampton to London and back again (400 km dispersion-compensated transmission distance, 6 inline flat-gain EDFAs with maximum input/output powers of $-5 / 15 \mathrm{dBm}$, operated in automatic gain control mode with a nominal gain of $20 \mathrm{~dB}$ ). The maximum total power launched into the link was $7 \mathrm{dBm}$ with a maximum power along the link of $15 \mathrm{dBm}$. The detailed configuration is shown in [58]. At its output, ITU Channel 27 was converted to Channel 23 [Copy -1] (either with or without regeneration) and sent through the link again together with all the remaining channels. A total of 38 channels occupied the frequency grid at any time. 


\section{FIGURE 16 GOES HERE}

The regenerator was mounted into a standard telecommunication rack along with other network components and test gear. To enable a comparative study with/without regeneration, conventional wavelength conversion could be carried out by switching off Pump 2, resulting in phase insensitive FWM-based wavelength conversion.

\section{FIGURE 17 GOES HERE}

Results obtained after $800 \mathrm{~km}$ with the regenerator used "in-line" after $400 \mathrm{~km}$ are shown in Figure 17. Use of a mid-point regenerator was capable of reducing the BER power penalty by a factor of two (e.g., at $\mathrm{BER}=10^{-9}$, from $5 \mathrm{~dB}$ to $2 \mathrm{~dB}$ ). The regenerator also lowered the error floor by one order of magnitude, Figure 17a. Varying the input power into the link, Figure 17b, showed almost $4 \mathrm{~dB}$ power penalty in the second round trip. This value was reduced to $2 \mathrm{~dB}$ when the regenerator was used.

\subsection{Analog Error Correction in PSA Regenerators}

\section{FIGURE 18 GOES HERE}

PS regenerators can correct errors when differential decoding of PSK is performed. This is counterintuitive as regenerators typically do not improve BER when placed in front of a receiver. This phenomenon is due to a correctable class of errors specific to a 'differential' receiver. Consider an example where two consecutive bits with identical phases of $\pi$, Figure 18, which during transmission accumulate phase errors of $\pi / 3$ and $-\pi / 3$, respectively. A DPSK receiver detects the difference in the phases of these consecutive bits as $2 \pi / 3$. The receiver's decision point is set at $\pi / 2$ resulting in an erroneous detection of $\pi$ as the differential phase of the two bits rather than the correct value of 0 . On the other hand, a PSA corrects the phase of each bit, before the evaluation of their difference in the differential receiver, so such an error would not occur. This ability to correct for differential errors means that after the PSA process the absolute and differential phase noise exhibit almost the same statistics for signals degraded by nonlinear phase noise [57]. This suggests that a DPSK receiver supported by a PSA pre-amplifier could perform as well as an ideal homodyne PSK receiver. Therefore, in principle a homodyne coherent receiver, which is usually implemented with power greedy digital electronics, could be substituted by a simpler DPSK receiver assisted by a PSA regenerator.

\section{MPSK Phase Regeneration}

Conventional PSA based schemes are incapable of directly supporting advanced formats such as quadrature phase shift keying, whose four phase states allow two bits per transmitted symbol. To achieve regeneration of signals with more than two levels, a number of indirect approaches have been suggested. One of these is the use of parallel BPSK regenerators, each regenerating one of the I and Q quadratures in a QPSK signal [59]. Another solution is to perform BPSK to OOK conversion using linear interferometers, to amplitude regenerate the OOK streams in parallel, and then to perform alloptical format conversion to synthesize a clean (regenerated) QPSK signal [60, 61]. The parallel approach of both these schemes, often favoured in electronics, is complicated in optics due to the requirement to equalize and stabilise multiple optical paths (often over $100 \mathrm{~m}$ in length with silica fibre based implementations) in phase, polarization and propagation delay, which is both complicated 
and costly. In addition, linear scaling of component count and power utilisation would offset much of the benefit of adapting higher order modulation formats.

In addition to regeneration, for more sophisticated processes such as analog-to-digital conversion (ADC), quantization to more than two levels is an absolute requirement. Research into photonic quantizers for ADCs has been ongoing for four decades. Whilst numerous architectures have been proposed [62], a niche for an ultrafast all-optical quantizer still exists. Photonic quantizers providing bandwidths $>100 \mathrm{GHz}$, effective number of bits (ENOB) of at least 4 and power consumption under $5 \mathrm{~W}$ would offer real competitive advantage as compared to the current electronic alternatives [62, 63]. With this in mind, effort was made to realise a scheme to quantize phase encoded signals to multiple levels.

\subsection{PSA-based Multilevel Phase Regeneration}

\subsubsection{Multilevel Phase Quantization Concept}

By developing a more general understanding of how phase can be manipulated, it is possible to derive schemes by which more sophisticated phase transfer functions can be synthesized. A simple way to do this is by taking the cosine function as an example. For an input $\phi$ ranging from 0 to $2 \pi, \cos (\phi)$ resembles a 2 level phase quantization operation as its result is a variable of phase $\phi_{s}=0$ for $\pi / 2 \leq \phi<3 \pi / 2$, and $\phi_{s}=\pi$ everywhere else. $\cos (\phi)$ can be re-written as the sum of complex exponentials obtaining

$$
\begin{aligned}
& \left|A_{s}(\varphi)\right| \cdot \exp \left(i \varphi_{s}\right)=\exp (i \varphi)+\exp (-i \varphi)=2 \cos (\varphi)= \\
& \left\{\begin{array}{c}
|2 \cos (\varphi)| \cdot \exp (i \cdot 0), \quad \text { if } \frac{\pi}{2} \leq \varphi<\frac{3 \pi}{2}, \\
|\cos (\varphi)| \cdot \exp (i \cdot \pi), \quad \text { elsewhere }
\end{array}\right.
\end{aligned}
$$

where $\phi_{s}$ is the 2-level quantized phase output, and $A_{s}(\phi)$ is the accompanying $\pi$-periodic amplitude response.

Equation 1.17 suggests that an arbitrarily phase encoded signal $\exp (i \phi(t))$ can be quantized in phase to 2 levels by coherently adding to it a phase conjugated version of the input signal, $\exp (-i \phi(t))$. This is in fact what happens in a degenerate PS-FOPA, as can be seen in Eqn 1.11.

The real leap forward enabling multi-level phase quantization is the realization that Eqn 1.17 can be generalised by rewriting it as [13]

$$
A_{s}(\phi) \cdot \exp \left(i \phi_{s}\right)=\exp (i \phi)+\frac{1}{M-1} \exp (-i \phi(M-1))
$$

where $M$ refers to the number of phase levels. Figure 19 shows the result of evaluating Equation 1.18 for 3 values of $M$. For $M=2$, the phase transfer function is very sharp, with a perfect $\pi$ step. This is accompanied by a strong phase-to-amplitude conversion. As the value of $M$ increases, the periodicity of the phase transfer function (TF) matches $M$ as expected, but the step becomes less defined, and the depth of the phase-to-amplitude conversion is also seen to reduce.

\section{FIGURE 19 GOES HERE}


The phase transfer functions as derived in Equation 1.18 are all monotonic with $M$ turning points. While this provides maximum local flatness at $0,2 / M \pi 3 / M \pi$ etc., it is not necessarily ideal for a quantizer in which the target is to minimise the global phase error variance. One solution is to rewrite Eqn 1.13 by substituting the coefficient of the conjugate term with a variable $m$,

$$
A_{s}(\phi) \cdot \exp \left(i \phi_{s}\right)=\exp (i \phi)+m \cdot \exp (-i \phi(M-1))
$$

Figure 20 shows the result of varying $m$ in Eqn 1.19 for $M=4$. Three values are used, including 0.33 , which is the coefficient as calculated from Eqn 1.18. To optimise for $m$, a misfit factor was designed such that an ideal value can be numerically identified. The misfit factor, as shown in Eqn 1.20, integrates the difference between a given phase transfer function, and an ideal step, hence the smaller the number achieved the better the quantizer.

$$
\text { MisfitFactor }=\log \left(\frac{2}{\pi} \int_{-\pi / M}^{\pi / M}\left|\phi_{s}(m, \phi)\right| \quad d \phi\right)
$$

The impact of varying $m$ on the misfit factor is shown in Figure 21 for $\mathrm{M}$ values of 2 to 5 . A misfit factor of 0 is obtained for $m=0$ for all values of $\mathrm{M}$, indicating that no phase quantization is achieved, and that the output phase is identical to that at the input. The smaller the misfit factor, the closer the phase transfer function approaches an ideal step. Clearly, phase quantization is easily achieved for $\mathrm{M}=2$, at $m=1$. The optimal values of $m$ for higher values of $M$ depart somewhat from 1/(M-1). Also, as $\mathrm{M}$ increases, the degree of quantization achievable is compromised, as evidenced by the decreasing misfit factor. Note also that the minima are fairly broad, meaning that $m$ does not have to be set very precisely.

\section{FIGURE 20 GOES HERE}

\section{FIGURE 21 GOES HERE}

\subsubsection{Multilevel Phase Quantizer Implementation}

The advantage of the exponential notation in Equation 1.14 is that it can be immediately deduced how to phase quantize to $M$ levels an arbitrary analytical phase encoded signal, $\exp (i \phi(t))$. This can be done by coherently adding to the signal a conjugated phase harmonic bearing a temporal phase modulation M-1 times that on the input [13]. Provided that the conjugation mechanism used is much faster than the data modulation (this criterion is met by utilising the ultrafast Kerr effect), $\phi$ in Eqn 1.14 can be replaced by a time-varying phase modulation, $\phi(t)$. The question is then how to synthesize, from an input phase modulated signal, the M-1th phase harmonic, conjugate it, scale it by $m$, and coherently add it to the input.

The functions of phase multiplication, conjugation and coherent addition can be performed using FWM as shown in Figure 22. First, the phase harmonic is generated from the signal using a cascaded FWM process (Figure 22a). This is done by combining the signal with a strong pump beam at a frequency detuning $\Delta f$ in a nonlinear medium. By optimising the phase matching and the strength of the nonlinear interaction, a spectral cascade of FWM products are generated (Figure 22a). Because FWM is momentum conserving, the comb of products possesses an overlying phase modulation that 
is a perfect integer multiple of the modulation present on the signal at the mixer input. Next, a second FWM process is carried out using two pumps located symmetrically around the signal and phase harmonic (Figure 22b) to coherently conjugate and add the M-1th phase harmonic to the signal. FIGURE 22 GOES HERE

\subsubsection{Inline PSA QPSK Regenerator}

As discussed in section 5.1.2, a single non-degenerate PSA can be directly used for MPSK regeneration provided that the input signal is coherently added to a conjugated phase harmonic (idler) bearing a temporal phase modulation M-1 times that on the input. Figure 22 shows how this can conceptually be achieved using FWM in a two-step process. In [64] this was demonstrated using two nonlinear fibres (one for each step). First, the QPSK signal, $\phi(t)$, was mixed with a pump to generate a FWM comb, including the required idler, $3 \cdot \phi(t)$. The signal and conjugated harmonic were then coherently added using a dual pump non-degenerate PSA. The first of the two PSA pumps was derived from the free-running laser used in the comb generation stage, and the second by injection locking the fourth FWM harmonic $(4 \cdot \phi(t)$, which is modulation stripped for a QPSK input) to a semiconductor laser, satisfying the phase locking requirement. The relative powers of the signal and harmonic needed to be optimised to take the PSA power gain $G$ into account; as such a signalharmonic offset of $m_{\text {eff }}$ was used, where $m_{\text {eff }}=m * \sqrt{1-1 / G}$. Absolute phase deviations of up to 60 degrees per symbol (peak-to-peak) for a QPSK input at 10 Gbaud were reduced by a factor of 2 [64].

In the interests of brevity that experiment is not described in detail here, but rather a modification to the setup that allowed a single HNLF to be used $[13,65]$ is presented. The two stage MPSK regenerator [64] is defined by the distinction between the first HNLF in which the phase multiplication and pump recovery occurs, and the second HNLF in which the conjugated idler is coherently added to the signal, hereby regenerating the phase. An interesting variant of that scheme was discovered $[13,65]$ in which phase sensitive gain is obtained directly from a $2 \mathrm{P}$ non-degenerate parametric amplifier without an idler at the amplifier input, in apparent contradiction to the expected characteristics of a non-degenerate PSA. This device configuration, in addition to allowing QPSK phase regeneration in a simpler configuration relying on just one nonlinear element, provided significantly enhanced amplitude noise improvement.

The concept is in effect a fusion of the two separate parametric effects into one distributed action. Provided that the two pumps 1 and 2 as well as the signal are present at the mixer input, the coherent mixing process can then be distributed along the fibre length; initially the cascaded mixing process dominates, and then as the harmonic idler starts to grow in power the coherent conjugated addition takes place. In $[13,65]$, this was experimentally demonstrated.

\section{FIGURE 23 GOES HERE}

The output of a CW laser at $1555.7 \mathrm{~nm}$ was split into two, with one portion coupled into a $10 \mathrm{GHz}$ comb generator, see Figure 23. Comb lines at -190 and $+570 \mathrm{GHz}$ detuning were injected into semiconductor lasers, providing two pump beams phase locked to the signal carrier. The rest of the signal light was modulated with a PRBS to generate single polarization QPSK. This was sent through a noise additive module to emulate the effects of linear (related to quantum noise and ASE) and nonlinear phase noise (related to nonlinear amplitude to phase conversion). This module (shown as an inset in Figure 23) comprised an ASE source whose output was split into two, one portion being detected and the resulting electrical white noise being used to drive a $\mathrm{LiNbO}_{3}$ phase modulator 
through which the signal was passed, and the other portion being optically combined with the signal in a coupler.

The signal was then combined with the pumps and all the waves were amplified in an EDFA, leading to $50 \mathrm{~mW}$ of signal power and $250 \mathrm{~mW}$ power per pump. They were then sent into an HNLF (OFS, Denmark). The HNLF parameters were length $300 \mathrm{~m}$, nonlinear coefficient $11.6 / \mathrm{W} / \mathrm{km}, \mathrm{ZDW} 1553$ $\mathrm{nm}$ and $1550 \mathrm{~nm}$ DS $0.018 \mathrm{ps} / \mathrm{nm}^{2} / \mathrm{km}$. It also had a strain gradient to increase its SBS threshold, allowing the use of continuous wave pumps. Slow thermo-acoustic relative phase drifts were suppressed by monitoring the signal power at the PSA output and controlling the PZT. The signal was then assessed using an EXFO constellation analyzer (PSO-200) based on all-optical sampling capable of operation up to 100 Gbaud.

The input and output spectra to the PSA are shown in Figure 24 and Figure 25 respectively. At the input to the PSA, there is a very weak component at the idler frequency ( $+380 \mathrm{GHz}$ detuning) emanating from weak FWM in the high power EDFA, but at $-40 \mathrm{~dB}$ relative to the signal this does not affect the subsequent parametric interaction as verified by numerical simulations. $7 \mathrm{~dB}$ phase sensitive gain variation was obtained at the PSA output as measured with the feedback to PZT turned off. The spectrum at the output to the PSA Figure 25 suggests two separate interactions occur simultaneously. Firstly, the presence of the strong component at $+190 \mathrm{GHz}$ detuning indicates coherent phase multiplication via mixing of the signal with the pump at $-190 \mathrm{GHz}$, and secondly, the strong idler at $+380 \mathrm{GHz}$ indicates conventional 2P phase insensitive amplification. There are many other parametric interactions that occur due to the strength of the signal and pumps, as shown in the wideband output spectrum, Figure 26. These extra mixing products can be used to enhance the functionality of the device, such as being used as a wavelength multicaster by accessing the wavelength-translated copies of the input signal. It could however be viewed as being energetically inefficient, but this transfer of energy to unwanted frequencies can be minimised by careful selection of the fibre parameters.

FIGURE 24 GOES HERE

FIGURE 25 GOES HERE

FIGURE 26 GOES HERE

To characterise the regenerator, signal constellations including data on the phase error variance and normalised variance of amplitude noise are shown in Figure 27 [65]. While this statistical information would be more robust if obtained from a homodyne receiver without digital phase compensation for the intradyne local oscillator, it is still useful for quantifying the relative signal improvements derived from the regenerator.

FIGURE 27 GOES HERE

The regenerator was first assessed at 10 Gbaud with added phase noise only, emulating the nonlinear regime. Pseudo-Gaussian phase fluctuations would be expected at the input, with an artificial roll-off at the tails of the distribution due to saturation of the photodiode for high ASE levels. The regenerator desirably reduced the phase error variance by a factor of 6 , while the amplitude noise variance only increased by 2.6 Figure 27, Cell A), suggesting an overall benefit as the input BER is dominated by the phase noise. This phase noise reduction is comparable to the numerically predicted factor of 5.5 for the parallel BPSK regenerator scheme [59], denoting that the inline compactness of the approach does not come with an associated performance penalty. For QPSK, a comparison with studies on the impact of phase estimation errors on BER suggests that the output phase error variance of $0.0042 \mathrm{rad}^{2}$ 
(Figure 27, Cell A) approximately corresponds to an SNR penalty under $0.5 \mathrm{~dB}$ for a BER of $10^{-4}$, while the input variance corresponds to a penalty of approximately $4 \mathrm{~dB}$ [66]. This nonlinear phase noise reduction implies that i) the reach of the transmission span can be increased ii) the tolerance to nonlinearity is significantly enhanced; hence higher receiver OSNRs can be envisaged.

In the presence of linear noise emulated by ASE loading (hence degraded OSNR with both phase and amplitude fluctuations), the phase error variance and normalised amplitude variance are simultaneously reduced by approximately 3.2, indicating even better net regenerator performance (Figure 27, Cell B). This ability to concurrently reduce both absolute phase and amplitude variance prior to the receiver suggests that the regenerator should provide significant signal quality improvement if deployed before a differential receiver, which normally requires a trade off between reduced complexity and lower noise tolerance compared to a fully coherent one.

The symbol rate was increased to 56 Gbaud and the measurements repeated. Without added noise, the regenerator preserves the phase quality at the expense of some amplitude noise (Figure 27, Cell C). It is believed that this is a result of the wideband parametric interactions occurring in the PSA, as shown in Figure 26, that transfer some amplitude noise to the signal. In the presence of phase noise, the phase error variance is reduced by a factor of 3.2, with increased amplitude noise at the output (Figure 27, Cell D). For QPSK however, in which the information is solely contained in the phase, this increased nonlinear phase noise tolerance would translate to increased reach or higher signal launch powers to improve OSNR at the receiver.

\subsection{Other approaches to QPSK regeneration}

\subsubsection{Interferometric (Parallel PSA) QPSK Regenerator}

\section{FIGURE 28 GOES HERE}

Zheng et. al. [59] proposed a technique that utilises two parallel PSAs to separately regenerate the I and Q quadratures of an input BPSK signal. These regenerated streams are then combined in parallel in a coupler, thereby re-synthesizing a cleaner QPSK signal. This concept has not been demonstrated experimentally, but numerical simulations showed the potential of the device to suppress phase noise significantly [59]. In principle, the performance of this device should be similar to that of the inline MPSK regenerator presented in Section 5.1.2, if correctly implemented. A similar scheme has been proposed by Da Ros et. al. [67] exploiting saturation effects in fiber optical parametric amplifiers using a pair of degenerate dual-pump PSAs. These interferometric schemes require one PSA per input quadrature, and therefore have double the component count for QPSK as compared to BPSK regeneration, in contrast to the inline approach.

\subsubsection{Interferometric format conversion based QPSK Regeneration}

A scheme utilising the separate regeneration of the I and Q quadratures was recently demonstrated by Kimchau et. al [61]. A $90^{\circ}$ optical hybrid with homodyne detection was used to convert the phase information of the I and Q components into four OOK signals that were then used to gate two alloptical wavelength converters based on semiconductor Mach-Zehnder interferometers, creating two phase coherent signals from a common local oscillator, each of which bears one of the I and Q streams. Combining these two BPSK signals in quadrature creates the regenerated QPSK signal. A 
major strength of this scheme is the visible route towards on-chip integration, although the demonstration itself included a separate EDFA for each of the $90^{\circ}$ optical hybrid's outputs. Note that implementing it requires the use of RZ input pulses.

\section{FIGURE 29 GOES HERE}

A similar scheme to this has been suggested by Matsumoto [6]. A DQPSK signal is converted to two parallel OOK signals by a pair of DLIs and the noise on the OOK signals is suppressed by all-optical $2 \mathrm{R}$ amplitude regenerators. The regenerated OOK signals are used as control pulses for a pair of alloptical phase modulators to generate phase-remodulated output pulses. This type of regenerator converts the phase difference between consecutive pulses in the input signal to the absolute phase of the outgoing regenerated signal so that the phase data encoded on the regenerated signal is altered by the regenerator. The conversion of the phase data can be undone by suitable encoding or decoding in the electrical domain.

\section{FIGURE 30 GOES HERE}

It is worth noting that all these interferometric schemes rely on regenerating the I and Q quadratures separately. This means that as the input signal scales in complexity, for higher values of $\mathrm{M}$, the regenerator component count will scale linearly. Also, these schemes are only capable of operating on modulation formats that can be generated by summing BPSK signals, such as QPSK, while the inline approach can cope with any $M$ value, including odd numbers such as 3 and 5. This makes the latter approach more versatile and significantly easier to implement, as the component count is independent of the complexity of the input signal.

\subsection{Flexible MPSK Quantization}

The inline PSA based regeneration approach is inherently multi-format, as discussed in section 5.1. Reconfiguring the regenerator from QPSK to an alternate modulation format such as 8-PSK can be achieved simply by generating a broader FWM comb (by increasing the signal and pump powers), passively selecting the desired harmonic and tuning the injection locked laser to the corresponding frequency. This was experimentally demonstrated in [13].

\section{FIGURE 31 GOES HERE}

A narrow linewidth CW fibre laser (Rock laser, NP Photonics) at $1555.7 \mathrm{~nm}$ was split into two, with one portion coupled into a $10 \mathrm{GHz}$ comb generator. Two semiconductor lasers were injection locked to comb lines, one representing pump 1 at approximately $-200 \mathrm{GHz}$ detuning from the signal frequency and the other at a frequency (M-1)*200 GHz away, with the value of M depending on the modulation format being assessed. For both lasers the injected power was close to $-30 \mathrm{dBm}$. The rest of the signal light was modulated, either in a $\mathrm{LiNbO}_{3}$ phase modulator with information generated in a $12 \mathrm{GS} / \mathrm{s}$ arbitrary waveform generator, or in the case of QPSK, in a $\mathrm{LiNbO}_{3}$ Mach-Zehnder modulator (MZM) driven by an electrical PRBS pattern, the optical output of which was coherently multiplexed from BPSK to QPSK in a DLI.

The modulated signal was sent through a noise additive module to emulate the effects of linear (related to quantum noise and ASE) and nonlinear phase noise (related to nonlinear amplitude to phase conversion). The signal $(50 \mathrm{~mW})$ was then combined with the pumps (total power $500 \mathrm{~mW}$ ) 
and all the waves were amplified in a polarisation maintaining (PM) EDFA. Use of a PM EDFA ensured that the signal and pumps were co-polarised in the subsequent HNLF.

They were then sent into a strained HNLF whose parameters were length $300 \mathrm{~m}$, nonlinear coefficient $11.6 / \mathrm{W} / \mathrm{km}$, zero dispersion wavelength $1552 \mathrm{~nm}$ and $1550 \mathrm{~nm}$ slope $0.018 \mathrm{ps} / \mathrm{nm}^{2} / \mathrm{km}$. This had a strain gradient to increase its SBS threshold, allowing the use of CW pumps. Optical spectra at the input and output of the HNLF are shown in Figure $31 \mathrm{~b}$ and c respectively. Slow thermo-acoustic relative phase drifts were suppressed by monitoring the signal power at the PSA output and controlling the PZT. The processed signal was then combined with a local oscillator (tapped off the signal laser prior to data modulation) in a 90 degree hybrid followed by real-time $50 \mathrm{GS} / \mathrm{s}$ sampling of the two hybrid outputs detected in single ended fashion. Off-line post processing was used to retrieve the phase and amplitude of the signal.

To verify the operating principle, the signal phase was first varied over $2 \pi$ at a rate of $150 \mathrm{MHz}$. To switch to M-level quantization, the only requirement is to tune the frequency of laser 2 to $f_{s}+(M-1) \Delta f$, and optimise the pump and signal powers into the HNLF. This means that from a practical point of view, changing the modulation format or supported signal bandwidth (given by $\Delta f$ ) can be achieved in milliseconds or less without any hardware changes, relying on the rapid tuning capability of semiconductor lasers. The choice of signal and pump powers depends on the fibre parameters, M-level, and application, but as an example, $50 \mathrm{~mW}$ and $250 \mathrm{~mW}$ respectively proved ideal for QPSK regeneration.

\section{FIGURE 32 GOES HERE}

The signal can be represented in constellation diagrams, as shown in Figure 32. Quantization to 3, 5 and 6 levels was demonstrated. As expected, the quantization is accompanied by a sinusoidal amplitude response, whose depth decreases as $\mathrm{M}$ increases. If stronger phase squeezing was required, as might be for the higher levels of $\mathrm{M}$, multiple nonlinear elements can be cascaded in series. The quantization is accompanied by phase-to-amplitude conversion which would be undesirable for some applications; this however can be eliminated by subsequently regenerating the signal amplitude in a high dynamic range limiting optical amplifier, such as an injection-locked semiconductor laser [22]. Figure 33 shows the intermediate stages for 3- and 5-PSK, between the condition of zero pump power hence no nonlinearity, and the optimum pump power for ideal phase regeneration.

\section{FIGURE 33 GOES HERE}

\section{Choice of nonlinear materials and designs for all-optical signal processing}

The previous sections have reported on the substantial advances that have been achieved on the regeneration of phase encoded signals over the last few years. All of the demonstrations that we have reviewed employed silica-based HNLFs. While this technology arguably represents the most mature solution in terms of achieving highly controllable nonlinear optical effects, it is often desirable to opt for more compact nonlinear devices that lend themselves to easier sub-system integration. This section briefly reviews the features as well as some of the potential issues associated with each of the various material platforms that have been investigated for all-optical signal processing and the 
regeneration of phase encoded optical signals in particular. We start with an overview of the considerations that relate to $\chi^{(3)}$ based nonlinear systems, including highly nonlinear optical fibres (both in silica and non-silica glasses) and then present recent progress achieved in periodically poled lithium niobate (PPLN) and semiconductor optical amplifiers (SOAs).

\subsection{Third-order nonlinearity systems}

The observation of broadband nonlinear effects, and especially parametric effects such as those involved in OPAs, require a combination of both high nonlinearity and suitable dispersion characteristics. The design of optical fibres for nonlinear applications relies on engineering precisely the geometry and refractive index profile of the core-cladding region. It is then possible to attain designs that ensure a tightly confined guided field, thereby resulting in a high nonlinear coefficient, and that at the same time precisely manipulate the overall chromatic dispersion of the fibre. Germanium-doped silica fibre exhibiting exceptional dispersion properties and low transmission losses (around $1 \mathrm{~dB} / \mathrm{km}$ ) over a broadband wavelength range represents a well-established and reliable nonlinear platform [68]. However, their nonlinear coefficients typically range between $10-30 \mathrm{~W}^{-1} \mathrm{~km}^{-1}$, therefore in order for nonlinearities of sufficient strength to be observed at realistic power levels, long lengths (hundreds of meters) of fibre need to be used. The amount of launched power into such devices is ultimately restricted by the onset of SBS, which depletes the signal and effectively acts as a power limiter and noise source. The growth of SBS effects can be inhibited by broadening the SBS gain bandwidth and reducing the peak gain, by for example, applying a gradient either on the core diameter, the temperature or the strain of the fibre [69]. Whilst an order of magnitude increase in SBS threshold can be achieved in this way all of these techniques affect the chromatic dispersion uniformity along the fibre length which often significantly degrades device performance in other ways. Doping the core with aluminium [56, 68] which provides for low peak Brillouin gain coefficients has also been shown to be an effective technique, however this comes at the expense of a reduced nonlinear coefficient (typically $\gamma=7 \mathrm{~W}^{-1} \mathrm{~km}^{-1}$ ) and a higher attenuation (around $15 \mathrm{~dB} / \mathrm{km}$ ). Nevertheless various PSA demonstrations reported in the literature have already benefitted from the application of these techniques $[57,58,64,65]$.

More compact fibre-based solutions operating at $\mathrm{mW}$ power levels are possible by employing glasses with a higher nonlinear refractive index $\mathrm{n}_{2}$, such as bismuth oxide, lead silicate and chalcogenides. These glasses have invariably been used in either step-index [70-73] or photonic crystal fibre [74-76] designs, exhibiting up to three orders of magnitude higher nonlinearity than conventional silica singlemode fibres. Tapering of the waist diameter of such fibres has also been used to boost the nonlinear coefficient still further [77, 78]. Moreover, the relative strength of the Kerr to Brillouin nonlinearities in these glasses is much greater than in silica and consequently the maximum Kerr-induced nonlinear phase shift before the onset of SBS is often much higher than for silica-based HNLFs [70, 79], although in absolute terms the SBS threshold may be much lower). Fibres of this class have been used in the past for the implementation of short (a few meters long) reshaping regenerators for intensity modulated signals $[71,80]$, which can subsequently be used as the building blocks for indirect phase regeneration subsystems based on format conversion. Nevertheless, phase and amplitude regeneration in a FWM-based PSA has already been demonstrated in a 5.6m-long bismuth oxide HNLF $(\gamma=$ $\left.1100 /(\mathrm{W} \cdot \mathrm{km}), \mathrm{D}=-300 \mathrm{ps} /(\mathrm{nm} \cdot \mathrm{km}), \gamma \mathrm{P}_{\mathrm{SBSth}} \mathrm{L}_{\text {eff }}=1.1\right)$ [12], while using $1.56 \mathrm{~m}$ of a lead silicate fibre with significantly lower dispersion, phase sensitive amplification over a broad wavelength range has been demonstrated $\left(\gamma=820 /(\mathrm{W} \cdot \mathrm{km}), \mathrm{D}<-5 \mathrm{ps} /(\mathrm{nm} \cdot \mathrm{km}), \gamma \mathrm{P}_{\mathrm{SBSth}} \mathrm{L}_{\mathrm{eff}}>0.83\right)[81]$.

When it is important that the device length should be of the order of $\mathrm{cm}$, fibre solutions have to be abandoned. Indeed, when considering PSA applications for example, any device that could provide 
sufficient parametric gain could eventually be used for regeneration. Chalcogenide glasses have long been known for their high nonlinear refractive index [82] and recent results have shown their potential for the implementation of cm-long rib waveguides with a nonlinear coefficient of the order of $10^{5} \mathrm{~W}^{-1} \mathrm{~km}^{-1}$ [83]. Dispersion tailoring as required for different applications, as well as low propagation losses $(0.05 \mathrm{~dB} / \mathrm{cm})$ are also possible [84-86]. Silicon waveguides have also attracted significant interest recently, both because of their strong nonlinearities and their potential for further integration with electronic integrated circuits. Nonlinear waveguides have been demonstrated employing either pure silicon waveguide structures or silicon-organic hybrids, where the silicon waveguide is covered with organic nonlinear materials [87-89]. The cross-sectional dimensions of the waveguides can be of the order of tens of nanometers and the high optical confinement contributes to achieving an ultrahigh nonlinearity (up to $4 \times 10^{5} \mathrm{~W}^{-1} \mathrm{~km}^{-1}$ ) [90-92]. By appropriate engineering of the waveguide dimensions, the dispersion can be tailored to the desired values [79], and by adopting new fabrication processes relatively low losses $(4.5 \mathrm{~dB} / \mathrm{cm})$ are achievable. Impressive recent results have shown the potential of mm-long waveguide devices in ultrahigh-speed optical signal processing at data rates up to $1.28 \mathrm{~Tb} / \mathrm{s}$ at a relatively low operating power [92]. The amount of power launched into either chalcogenide or silicon waveguides is ultimately limited by the onset of two-photon absorption (TPA). In silicon in particular, where the effect is stronger, TPA is likely to be an issue for any applications operating below $2 \mu \mathrm{m}$.

Table 1 Summary of typical parameters for the $\chi^{(3)}$ based devices/materials discussed above. Two-photon absorption figure of merit: TPA FOM $=n_{2} /(\beta \lambda)$, where $\beta$ is the TPA coefficient. Stimulated Brillouin Scattering figure of merit: $\mathrm{SBS} F O M=\mathrm{P}_{\mathrm{SBSth}} \gamma \mathrm{L}_{\text {eff }}$, where $\mathrm{L}_{\text {eff }}$ is the effective length.

\begin{tabular}{|c|c|c|c|c|c|c|}
\hline Device and material & $\begin{array}{l}\text { Nonlinearity } \\
{[/ \mathrm{W} / \mathrm{km}]}\end{array}$ & $\begin{array}{l}\text { Dispersion } \\
{[\mathrm{ps} / \mathrm{nm} / \mathrm{km}]}\end{array}$ & $\begin{array}{l}\text { Loss } \\
{[\mathrm{dB} / \mathrm{m}]}\end{array}$ & $\begin{array}{l}\text { Device } \\
\text { length } \\
{[\mathrm{m}]}\end{array}$ & $\begin{array}{l}\text { TPA } \\
\text { FOM } \\
{[\mathrm{cm} / \mathrm{GW}]}\end{array}$ & $\begin{array}{l}\text { SBS FOM } \\
{[\mathrm{rad}]}\end{array}$ \\
\hline $\begin{array}{l}\text { Ge-Silica } \quad \text { HNLF } \\
{[68]}\end{array}$ & $10-30$ & $\begin{array}{l}\text { Selectable } \\
\text { close to } \\
\text { zero }\end{array}$ & $10^{-3}$ & $\begin{array}{l}100- \\
1000\end{array}$ & High* & 0.21 \\
\hline $\begin{array}{l}\text { Strained Al-Silica } \\
\text { HNLF [69] }\end{array}$ & 7.4 & $\begin{array}{l}\text { Selectable } \\
\text { close to } \\
\text { zero }\end{array}$ & 0.015 & $100-300$ & High* & 1.56 \\
\hline Soft glass fibres & & & & & & \\
\hline $\begin{array}{l}\text { Chalc. fibre taper } \\
{[77,78]} \\
\text { W-type index profile } \\
{[81]}\end{array}$ & $\begin{array}{l}68 * 10^{3} \\
820\end{array}$ & $\begin{array}{l}282 \\
\text { Selectable } \\
\text { close to } \\
\text { zero }\end{array}$ & $\begin{array}{l}18 \\
2.1\end{array}$ & $\begin{array}{l}0.22 \\
2\end{array}$ & $\begin{array}{l}2.83 \\
\text { High* }\end{array}$ & $\begin{array}{l}0.85 \\
>0.83\end{array}$ \\
\hline $\begin{array}{l}\text { Chalc. waveguides } \\
\mathrm{As}_{2} \mathrm{~S}_{3}[85] \\
\mathrm{Ge}_{11.5} \mathrm{As}_{24} \mathrm{Se}_{64.5}[86]\end{array}$ & $\begin{array}{l}10 * 10^{3} \\
136^{*} 10^{3}\end{array}$ & $\begin{array}{l}29 \\
66\end{array}$ & $\begin{array}{l}60 \\
260\end{array}$ & $\begin{array}{l}0.06 \\
0.018\end{array}$ & $\begin{array}{l}60 \\
60\end{array}$ & $\begin{array}{l}\text { Predict } \sim 1^{*} \\
\text { Predict } 1^{*}\end{array}$ \\
\hline $\begin{array}{l}\text { Si waveguides [79] } \\
\text { SOH waveguides } \\
{[87-89]}\end{array}$ & $\begin{array}{l}3 * 10^{5} \\
1 * 10^{5}\end{array}$ & $\begin{array}{l}\text { Selectable } \\
\text { close to } \\
\text { zero } \\
-7000\end{array}$ & $\begin{array}{l}400 \\
1500\end{array}$ & $\begin{array}{l}0.005 \\
0.004\end{array}$ & $\begin{array}{l}0.77 \\
2.19\end{array}$ & $\begin{array}{l}\text { Predict } \\
\text { High* } \\
\text { Predict } \\
\text { High* }\end{array}$ \\
\hline
\end{tabular}

${ }^{+}$Measured at $1545 \mathrm{~nm} .{ }^{*}$ No direct measurement found. 


\subsection{Quadratic nonlinearity systems}

A different approach to nonlinear processing involves the use of quadratic $\left(\chi^{(2)}\right)$ nonlinearities. In this case, the material of preference for telecommunications applications is lithium niobate, which can be periodically poled to form highly efficient nonlinear waveguides of a few centimeters length. Since most $\chi^{(2)}$ effects involve the generation of a second harmonic, it is necessary to employ cascaded nonlinearities in order to facilitate the use of pumps and signals in the same wavelength band. Such schemes typically use one pump operating within the phase-matched frequencies of the PPLN waveguide for the generation of the second harmonic, which in turn interacts with the signal via e.g. difference frequency generation. The application of cascaded quadratic nonlinear processes offers a number of potential advantages, such as broadband operation, low-latency, no intrinsic frequency chirp and SBS resilience [93-100]. However, such devices are ultimately limited by power transfer to the third harmonic wavelength, infrared absorption and subsequent temperature instability. A nondegenerate PSA was demonstrated in a PPLN waveguide using cascaded quadratic nonlinearities in [93]. Subsequently, this concept was also employed in [95] to demonstrate squeezing of the phase noise of a DPSK signal (Figure 34). Interestingly, this demonstration also considered the generation of the phase-locked signal-idler pair in a second PPLN waveguide, further miniaturizing the system. In a different application, phase-regenerative wavelength conversion was demonstrated in [94].

\section{FIGURE 34 GOES HERE}

\subsection{Semiconductor optical amplifiers}

Despite the advantages that PPLN-based devices offer both in terms of compactness and overcoming SBS issues, the pump power levels required for the operation of phase regenerators based on such devices, are still comparable to those of fibre-based systems. Semiconductor optical amplifiers (SOAs) on the other hand, are also promising active devices for signal processing as they have high integration ability and low switching energy [101-103]. By employing intersubband transition (ISBT) XPM effects [104] in quantum well or quantum dot material structures [102] SOA-based processing systems that combine a number of nonlinear devices on the same chip have already been demonstrated. In order to achieve operation at repetition rates exceeding 100Gbit/s the slow response component originating from the carrier energy relaxation and band-to-band carrier recombination can be solved using blue-wavelength filtering [105], or different configurations of symmetric-MachZehnder structures with SOAs in both arms [104, 106, 107]. However, a trade-off relationship between the optical power density required for absorption saturation and the relaxation time is intrinsic to this technology. The use of a monolithically integrated array of four SOAs inside two parallel Mach-Zehnder structures in a large-scale integrated circuit has been considered for indirect phase regeneration of DPSK signals based on format conversion [108]. Furthermore, the possibility of implementing the PSA operation in SOAs has also been investigated [109] and SOA-based signal regeneration (at least for the DPSK format) should be possible.

\section{Future trends and research directions}

So far this document has documented the remarkable progress that has been achieved in the regeneration of phase modulated signals over the last few years. It has to be appreciated however, that these advances represent only the first bold steps towards the implementation of all-optical processing systems that will address the networks of the future. In the paragraphs that follow, we will review early progress and challenges associated with two distinct directions, namely the simultaneous phase regeneration of more than one channel and the processing of signals modulated using more spectrally efficient modulation formats, such as quaternary amplitude modulation (QAM). 


\subsection{Multiwavelength regeneration}

In general, the multiwavelength operation of a nonlinear device is complicated, mainly since each of the co-existing signals can invariably act as a pump to the system. For example, the addition of an extra signal on a device based on $\chi^{(3)}$ nonlinearities can affect its response by the onset of additional effects originating by either cross-phase modulation, four-wave mixing or even Raman scattering. These impairments are less severe in cases where the signals are only required to propagate in a linear fashion within the nonlinear medium and share a common pump [14], or where the nonlinearities are localized within a certain wavelength region [110]. However, even in these cases, the presence of multiple signals drawing simultaneously from the power of the same pump can give rise to crosstalk. Despite this multi-channel PSA demonstrations based on a single pump non-degenerate parametric process have already been reported both in fibres and PPLN waveguides. In the PPLN-based demonstration reported in [110] one common pump was employed to effect phase squeezing of four independent channels through the use of cascaded quadratic nonlinearities. The experiment achieved squeezing of the standard deviation of the phase angle by up to 1.3 times, whereas the power of each of the four channels was $\sim 15 \mathrm{~dB}$ weaker than the pump to ensure that the parasitic cross-talk components were weaker than $22 \mathrm{~dB}$ below the generated signal-idler pairs. A fibre-based twochannel phase regenerative PSA based on a two-pump (per channel) degenerate parametric amplifier has also been presented recently (Figure 35) [111]. In this experiment, the two data signals shared the nonlinear medium and a common pump, whereas independent second pumps and phase-locking mechanisms for these pumps to each one of the signals were used. In order to avoid parasitic fourwave mixing interactions between the various pumps and signals, the signals were at least $10 \mathrm{~dB}$ weaker than each of the pumps, and care was taken to ensure that the frequency spacing between the two signals and the common pump was a non-integer multiple of the spacing between the two signals.

\section{FIGURE 35 GOES HERE}

The phase preserving amplitude regeneration scheme based on the application of a nonlinear amplifying loop mirror has also been extended to accommodate multiwavelength operation [112]. In this case, the approach taken was to demultiplex and employ a different nonlinear element per channel inside the loop mirror, thus allowing all other components of the regenerator to be shared between the different channels. Finally, a number of multi-channel intensity regenerators have been developed $[113,114]$ which can be used as building blocks in phase regenerators based on format conversion.

\subsection{Regeneration of other advanced modulation formats}

In general, the operation of signal regenerators relies on developing step-like transfer functions characterising the input and output parameters of interest (i.e. amplitude and/or phase). The majority of research on signal regeneration has been concerned with binary modulation formats requiring a single-stepped transfer function, whether this is in amplitude or in the $2 \pi$ phase cycle. Clearly, the processing of more complex modulation formats that encode the information in multiple levels of phase and/or amplitude require the engineering of increasingly more complex transfer functions. This was shown in Section 5 for the case of the QPSK format. The same section described the route towards using the principle of phase-sensitive amplification to implement transfer functions that satisfy a larger number of phase-only coding levels. This scaling of the PSA regeneration principle can satisfy certain useful modulation formats, such as 8-PSK. The regeneration of QAM signals in a single device would however require the implementation of both intensity and phase transfer characteristics that exhibit more than two levels. It has been shown that it may be possible to achieve 
intensity transfer characteristics that exhibit more than two levels and operate in a phase-preserving fashion [115] by extending the nonlinear loop mirror-based scheme of [19]. However, the regeneration of the complex field in both phase and amplitude and indeed in more than just two levels still represents a problem of considerable challenge. It is likely that the solution might lie in combining the respective techniques for phase and amplitude regeneration; however whether this route is feasible in practice, still remains to be seen.

\section{Conclusions}

In this Chapter we have reviewed the need, general principles and approaches used to regenerate phase encoded signals of differing levels of coding complexity and have described the key underpinning technologies used. We have discussed the evolution of the field and have presented the current state-of-the-art both in terms of the demonstrations of novel concepts that address and extend the range of functionality that can be achieved (e.g. range of advanced modulation format signals that can be addressed and new functions such as phase quantisation) and progress in terms of extending practicality (e.g. in terms of providing black-box operation and routes to integration). We have also given a personal perspective in terms of key issues and directions for future work.

From all that we have written it should be clear that this is a fast moving, exciting and potentially important research field with significant advances made in recent years, enabled in many instances by progress in the underpinning technologies, which include both highly nonlinear fibres and high performance narrow-linewidth lasers. It should however be equally clear that many challenges remain and that will need to be overcome if all-optical regeneration is ever to make it from the laboratory to commercial deployment in future optical networks - not least in terms of enabling significant levels of WDM capability and the development of compact, power efficient variants. Much work needs to be done and one can anticipate considerable innovation and interesting results ahead with many other application opportunities (e.g. within test and measurement systems) opening up along the way - we hope that this chapter encourages others to choose to enter and contribute to this interesting and potentially very important field. 


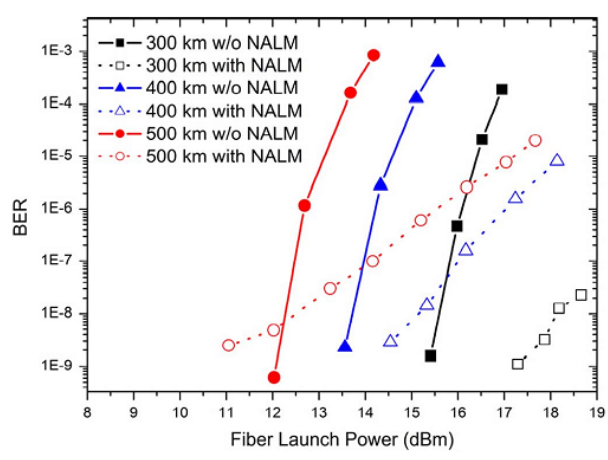

Figure 1: BER dependence of the fibre launch power for different transmission distances with and without a NALM regenerator every $100 \mathrm{~km}$, by Stephan et. al. (kindly reproduced from [20]).
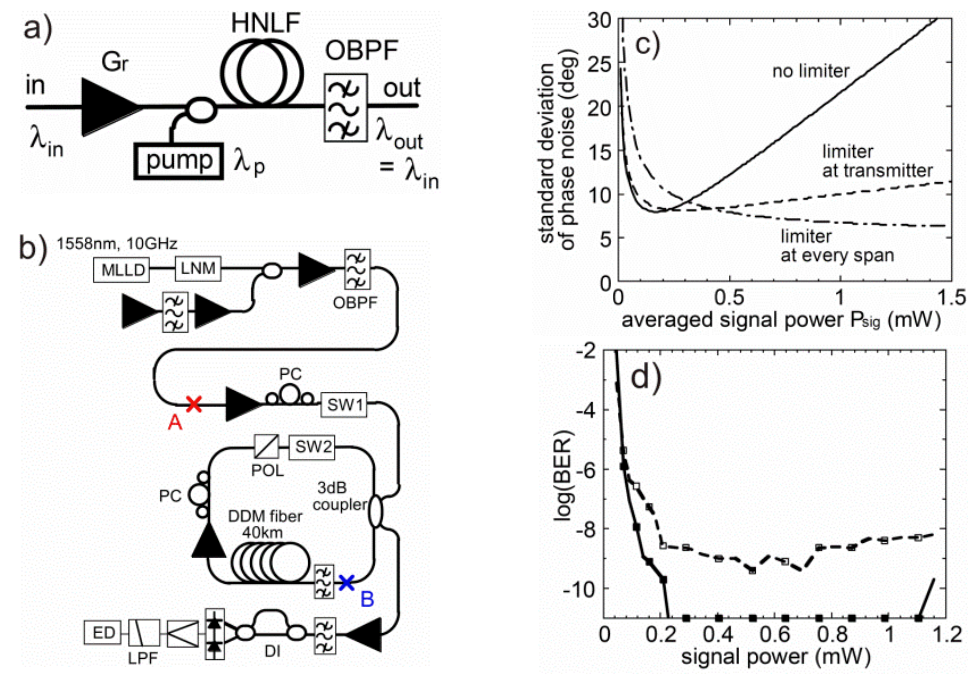

Figure 2: Phase preserving amplitude regenerator based on saturated FWM (a) and set-up of $10 \mathrm{Gbit} / \mathrm{s}$ short-pulse DPSK transmission using the regenerator (b). Comparison of performance when the regenerator was placed in the recirculating loop (point $B$ in (b)), or at a single position (point $A$ in (b)) is shown in (c). BER performance when regenerator in the recirculating loop (solid) and without the regenerator (dashed) is shown in (d). MLDD: mode-locked diode laser, LNM: modulator, SW1,2: acousto-optic switches, DI: delay interferometer, LPF: low-pass filter, ED: error detector (taken from [6]).

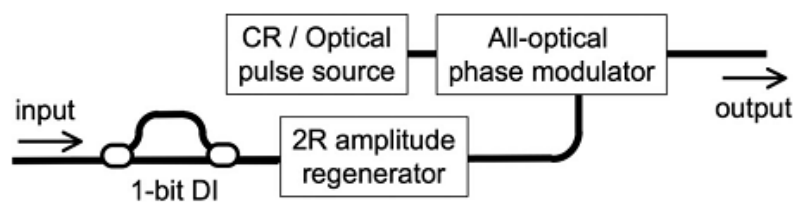

Figure 3: Block diagram of an all-optical DPSK signal regenerator using a straight-line phase modulator. CR: clock recovery circuit; DI: delay interferometer. Reproduced from [6]. 


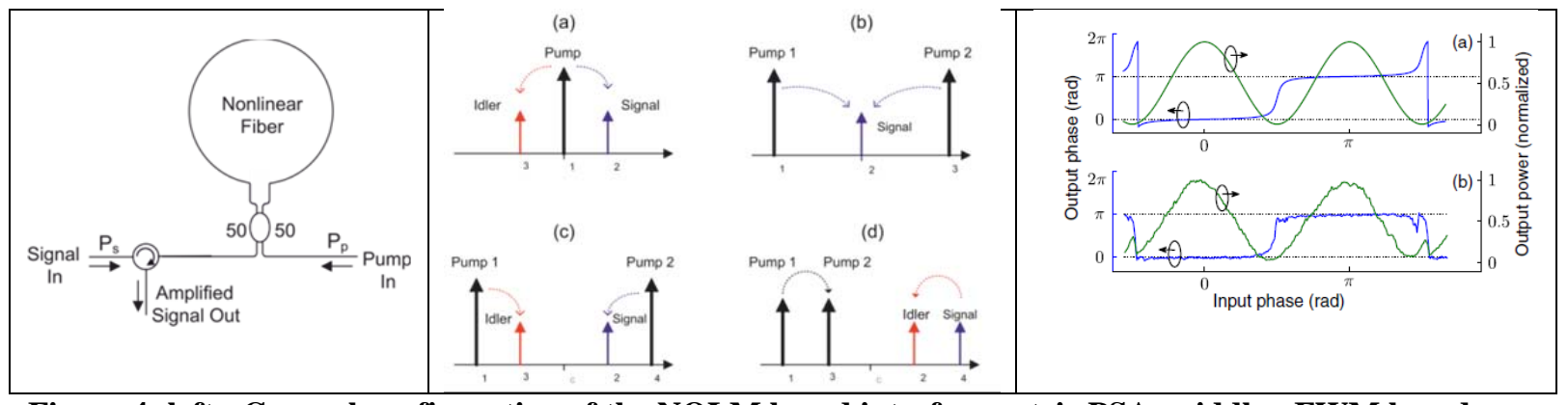

Figure 4: left - General configuration of the NOLM based interferometric PSA, middle - FWM based PSAs (a) single pump non-degenerate, (b) dual pump degenerate, (c) dual pump non-degenerate and (d) wavelength exchanger, right - (Color online) (a) Analytical and (b) experimental phase-to-amplitude and phase-to-phase transfer functions of a nondegenerate-idler PSA with a maximum of $11 \mathrm{~dB}$ of on-off gain, taken from [37].

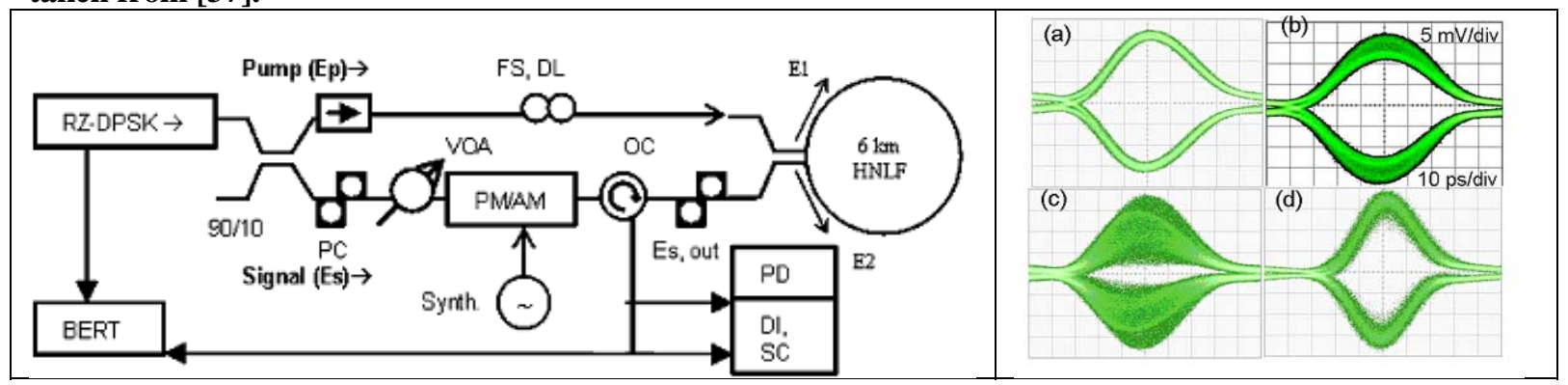

Figure 5: Experime5tal setup for RZ-DPSK regeneration (from [12]). FS: fibre stretcher; DL: delay line; VOA: variable optical attenuator; OC: optical circulator; PC: polarization controller; PD: photodiode; DI: delay interferometer; SC: sampling oscilloscope; Synth: synthesizer; BERT: bit-error-ratio tester; PM/AM: noise adding phase and amplitude modulators. Right: Eye diagrams after demodulation: (a) RZ-DPSK data, (b) With added AN, (c) With added PN and AN, (d) After regeneration [26].

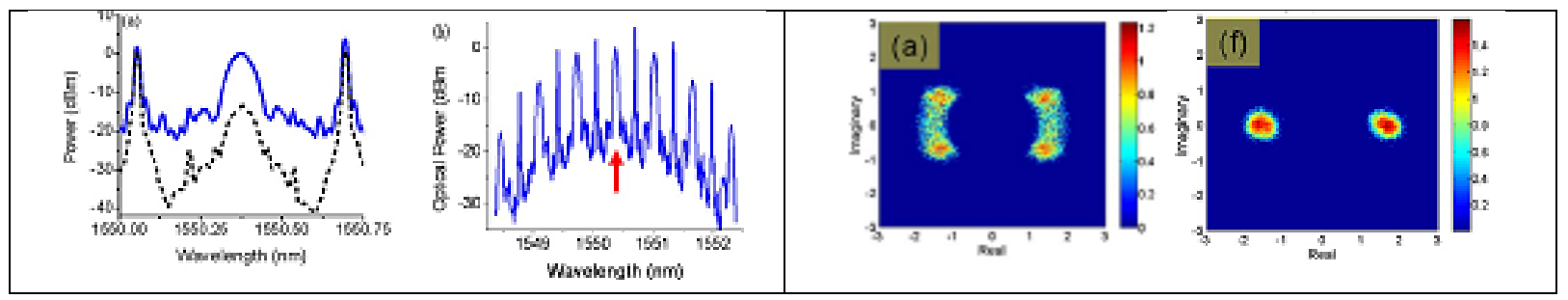

Figure 6: Left: Spectra for degenerate non-interferometric PSA with 80 GHz spaced pumps. (a) Spectrum before (dashed) and after (solid) PSA. (b) Full output spectrum: red arrow indicates location of the input signal. Right: Constellation plots of the input (a) and output (f) of the regenerator. Taken from [12].

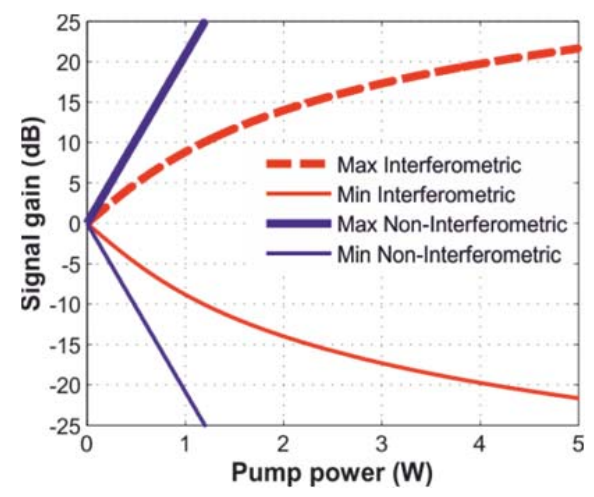


Figure 7: Analytic comparison (by simulation) of interferometric and non-interferometric PSAs using pump power and signal gain as a reference metrics.

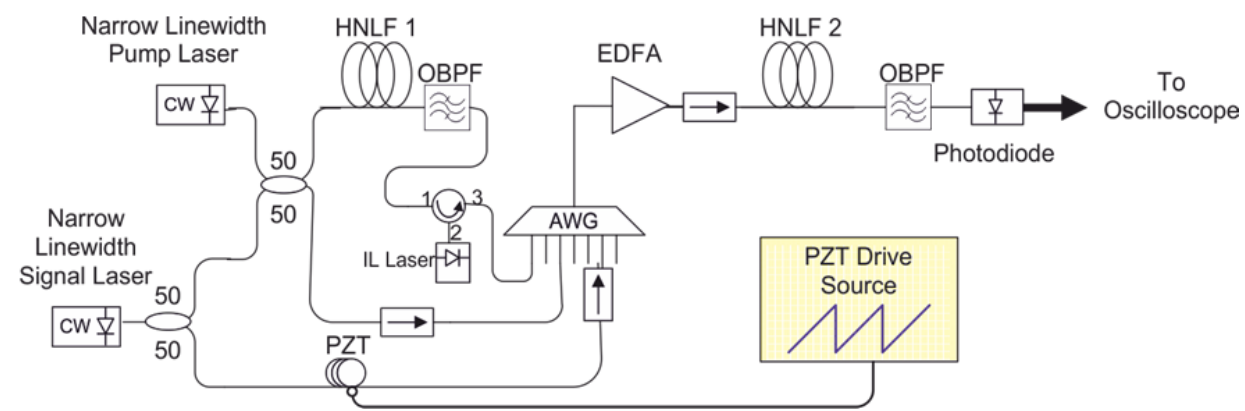

Figure 8 : Experimental setup for demonstrating saturation in dual pump degenerate PS-FOPA. OBPF: Optical Bandpass Filter [42].

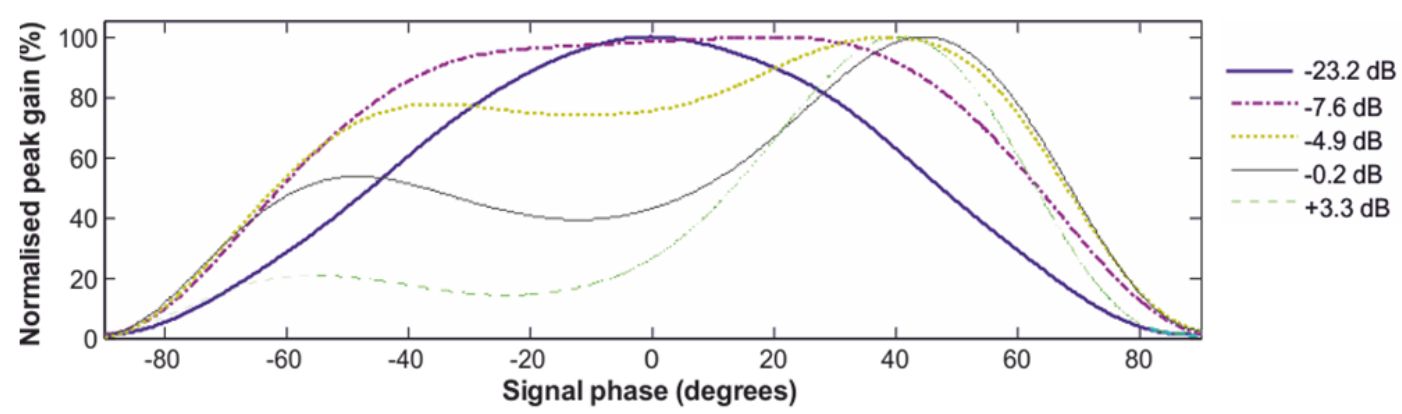

Figure 9: Phase-to-amplitude transfer characteristics for degenerate 2P PS-FOPA when signal power is boosted high enough to observe gain saturation [42].

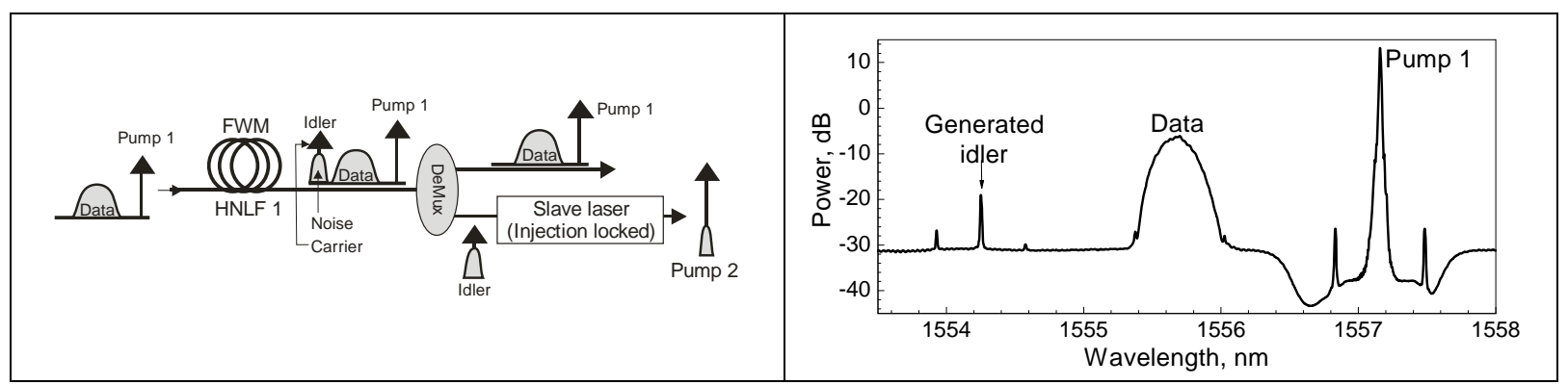

Figure 10: Phase synchronization of the pumps to the data signal. (a) Set-up; (b) Spectral characteristics at the output of HNLF 1.

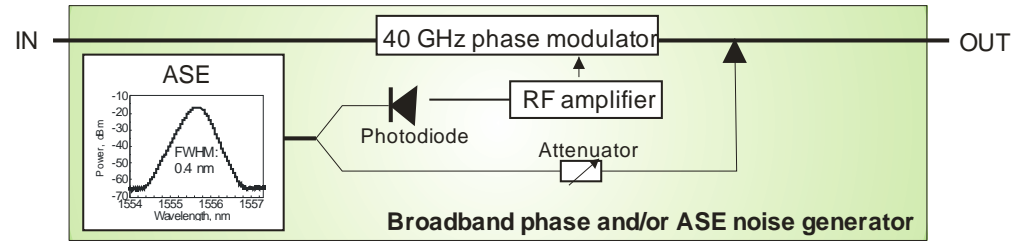

Figure 11: Noise emulator for adding linear noise (ASE) and non-linear noise (phase-only noise) via phase modulator driven by broadband RF noise generated using a fast photodiode detecting ASE noise. 


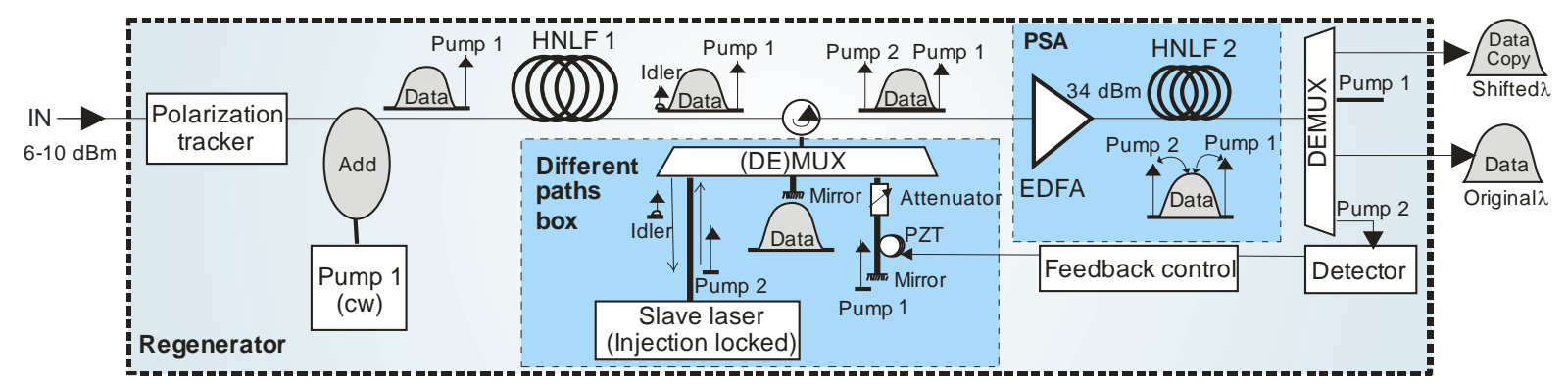

Figure 12: PSA regenerator set-up.

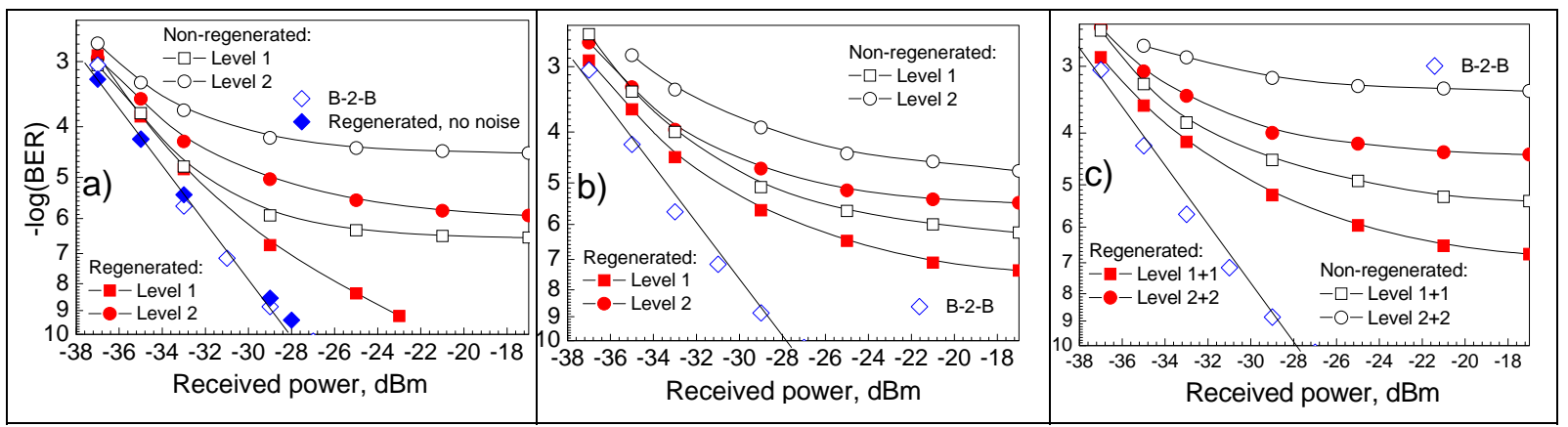

Figure 13: BER curves when phase-only noise (a), ASE-only noise (b) and phase+ASE noises (c) were applied at two different levels (from [57]).

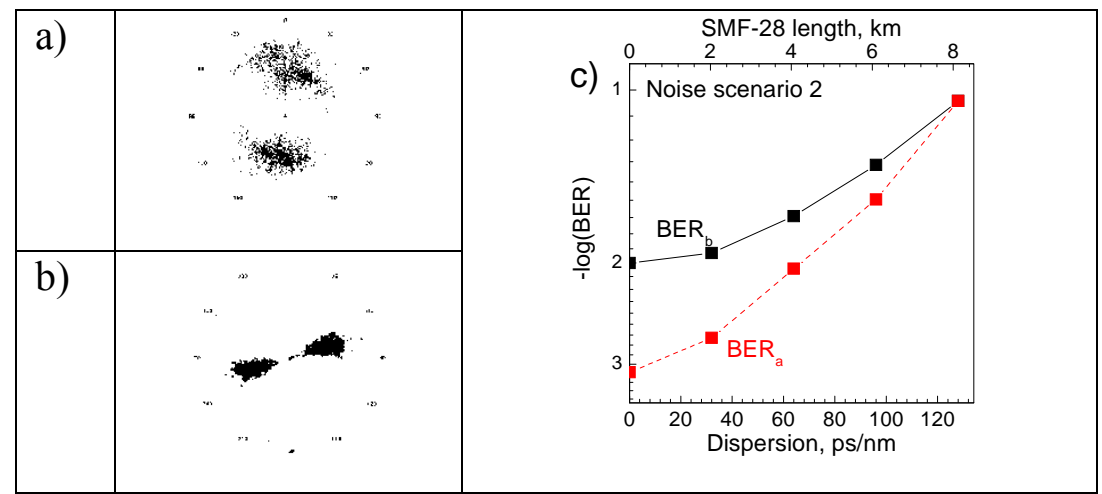

Figure 14: Calculated constellation diagrams of a $40 \mathrm{Gbit} / \mathrm{s}$ DPSK signal (a) before and (b) after the regenerator when signal has experiences residual dispersion of $80 \mathrm{ps} / \mathrm{nm}$. (c) Calculated BER as a function of the residual dispersion (from [58]).

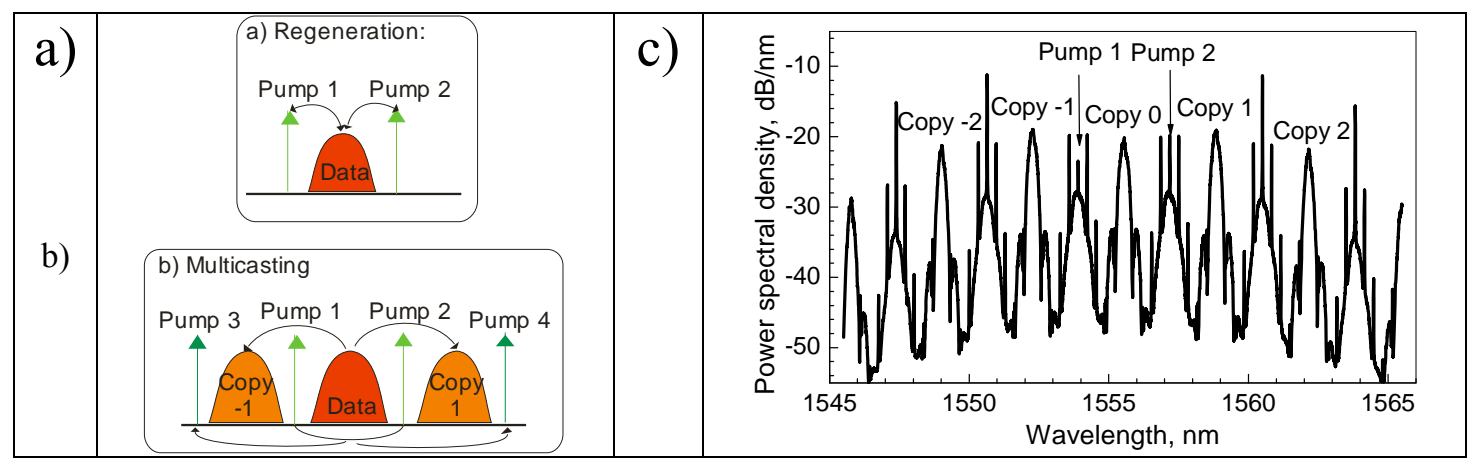

Figure 15: In an HNLF, several FWM processes are present such as (a) PSA and (b) generation of secondary pump and signal copies via phase insensitive FWM. Measured spectrum at the output of a HNLF as a result of such interaction is shown in c. In [57], the signal at the input signal wavelength (Copy 0) and four copies with power difference of less than $2 \mathrm{~dB}$ are considered. 

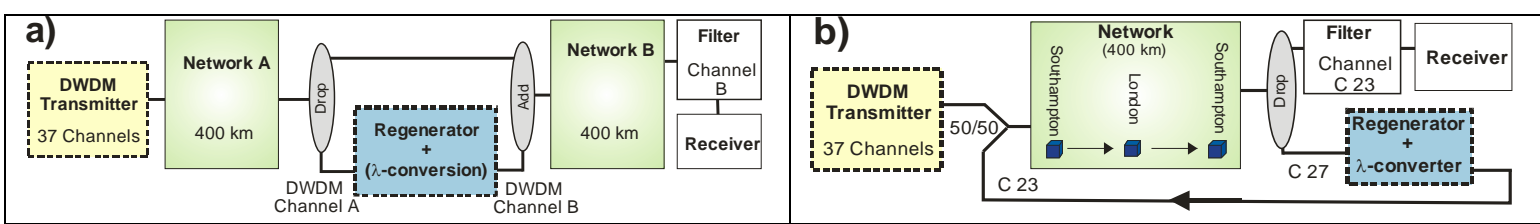

Figure 16: Model experiment with regenerator as an in-line device (a) and the implemented network that emulates key features of the model network (b).
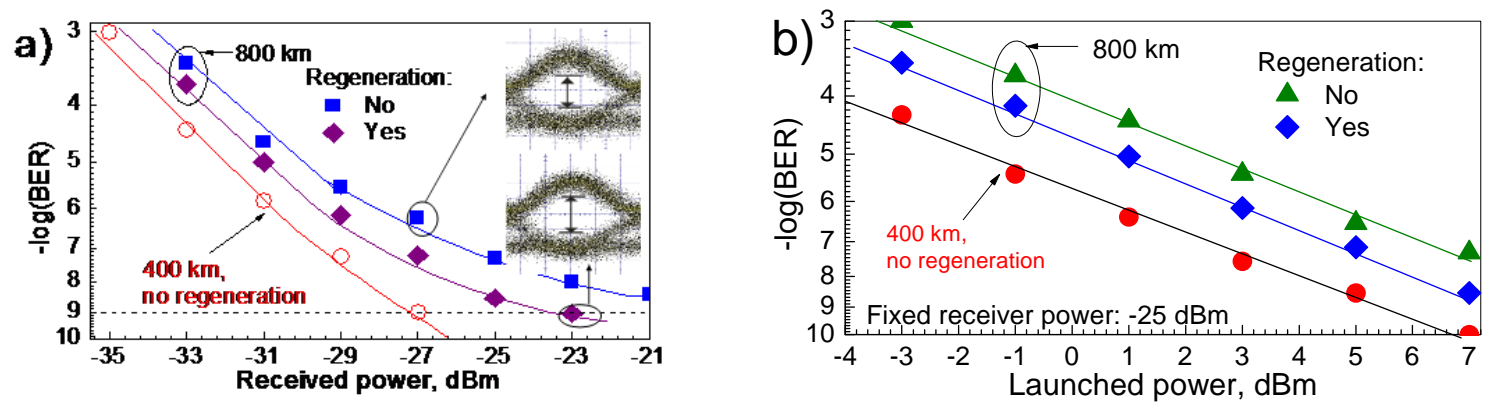

Figure 17: BER curves at the output of the $2^{\text {nd }}$ round-trip $(800 \mathrm{~km})$ with and without mid-span regeneration: (a) for launched power of $5 \mathrm{dBm}$; (b) as a function of the launched power for fixed receiver power of $-25 \mathrm{dBm}$. For reference, measurements of the signal at the mid-point (after wavelength conversion) are also shown.
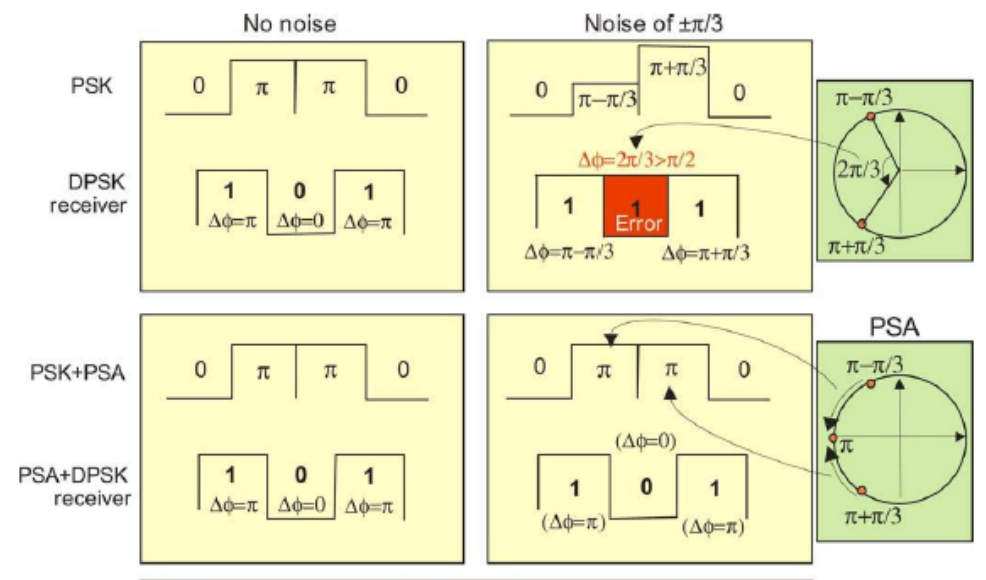

DPSK receiver: Logic $0:-\pi / 2<\Delta \phi<\pi / 2 \quad$ Logic 1 : $\pi / 2<\Delta \phi<3 \pi / 2$

Figure 18: Schematic explanation of the PSA for BER improvement in differential coherent (DPSK) receivers. A data sequence $0, \pi, \pi, 0$ is considered. 

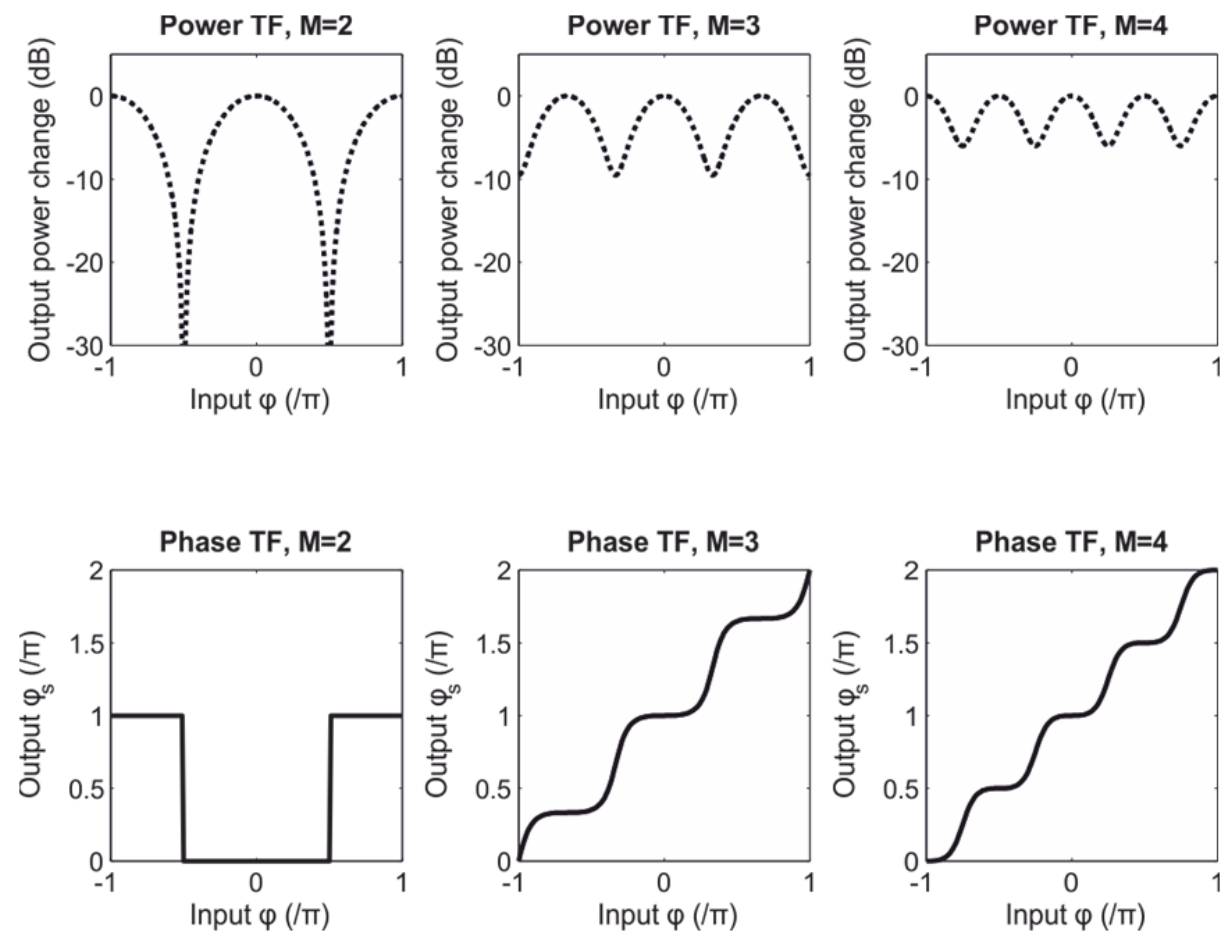

Figure 19: Evaluation of Eqn 1.13 showing how multilevel phase TFs are achieved as M is varied.
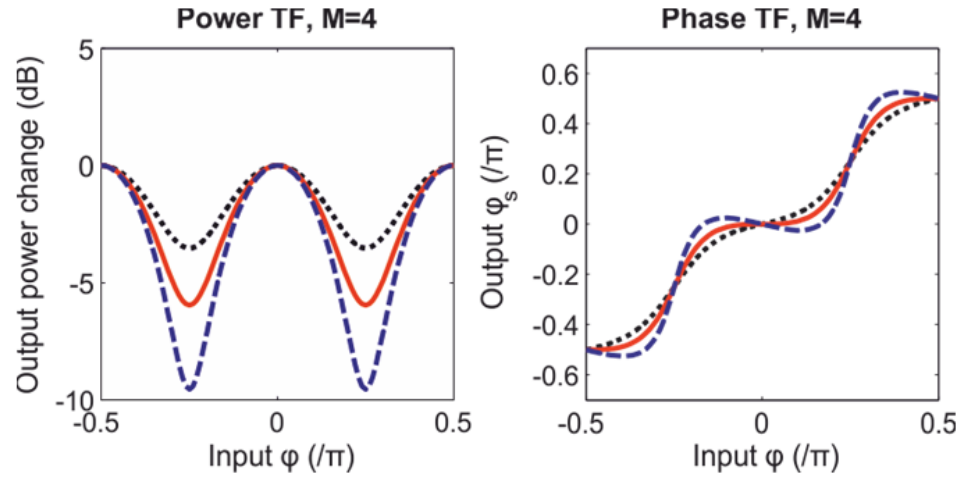

Figure 20: Evaluation of Eqn 1.14 for $M=4$, showing the transfer functions for various values of $\mathrm{m}$. Dotted line is for $\mathrm{m}=\mathbf{0 . 2 5}$, solid line $\mathrm{m}=\mathbf{0 . 3 3}$, and dashed line $\mathrm{m}=\mathbf{0 . 5}$.

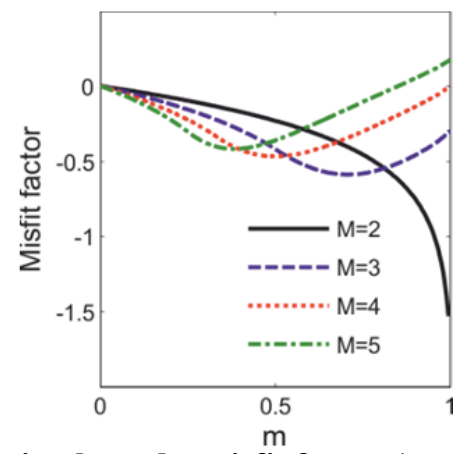

Figure 21: Evaluation of Eqn 6.3 showing how the misfit factor (as calculated in Eqn 1.15) as a function of $\mathrm{m}$ for various values of $M$. Optimum values for $M=2,3,4$ and 5 are $m=1,0.71,0.50$ and 0.38 respectively. 


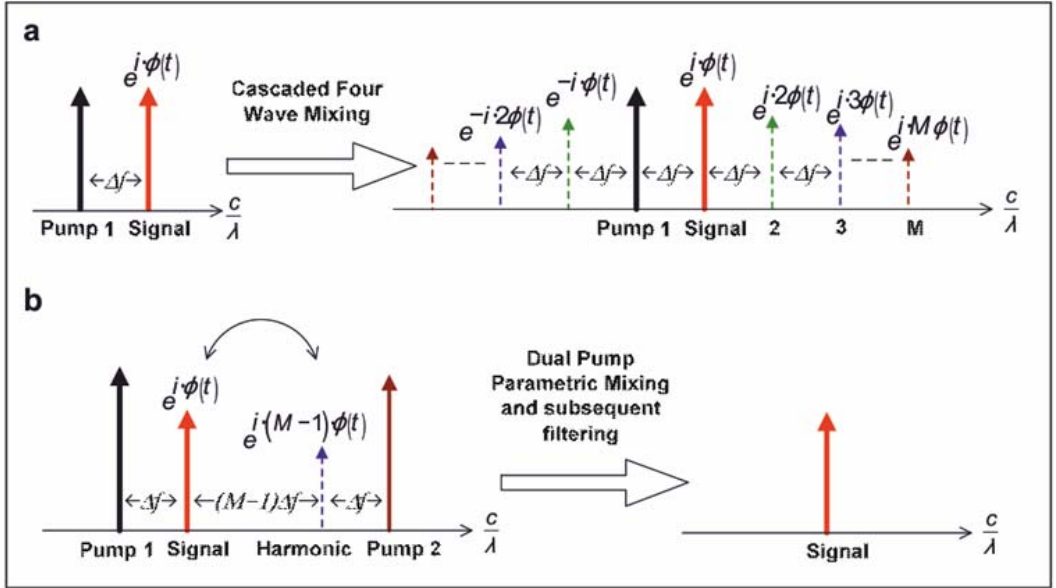

Figure 22: Illustration of how the M-level staircase transfer function necessary for phase quantization is achieved. a, By mixing a pump beam with a phase modulated signal in a nonlinear medium higher order phase harmonics of the signal can be generated. $b$, The signal is then coherently combined with the M-1th harmonic using a dual pump parametric process.

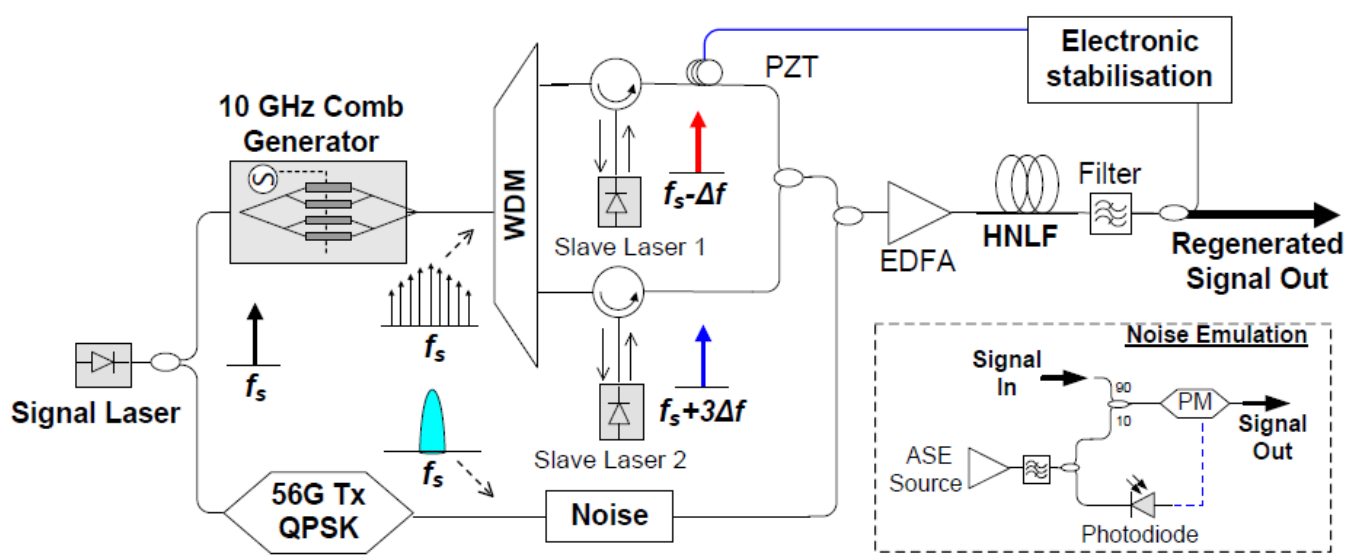

Figure 23: Regenerator setup, Tx - transmitter, WDM - wavelength division demultiplexer, PZT - piezoelectric fibre stretcher, PM - phase modulator [65].

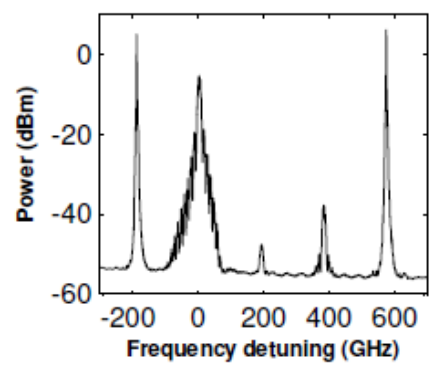

Figure 24: PSA input spectrum, signal located at 0 GHz detuning. 


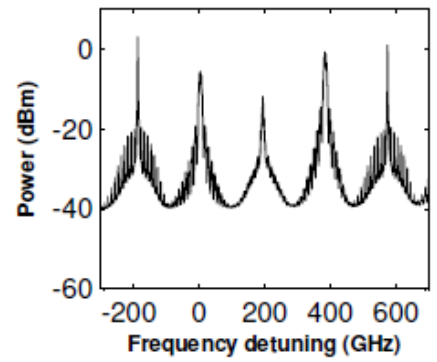

Figure 25 : PSA output spectrum, signal located at 0 GHz detuning, output idler.

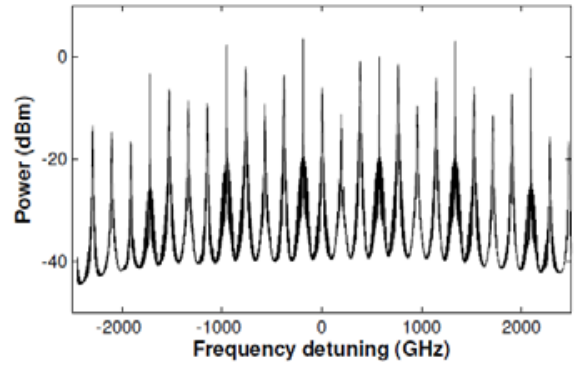

Figure 26: PSA output spectrum showing wideband mixing products.

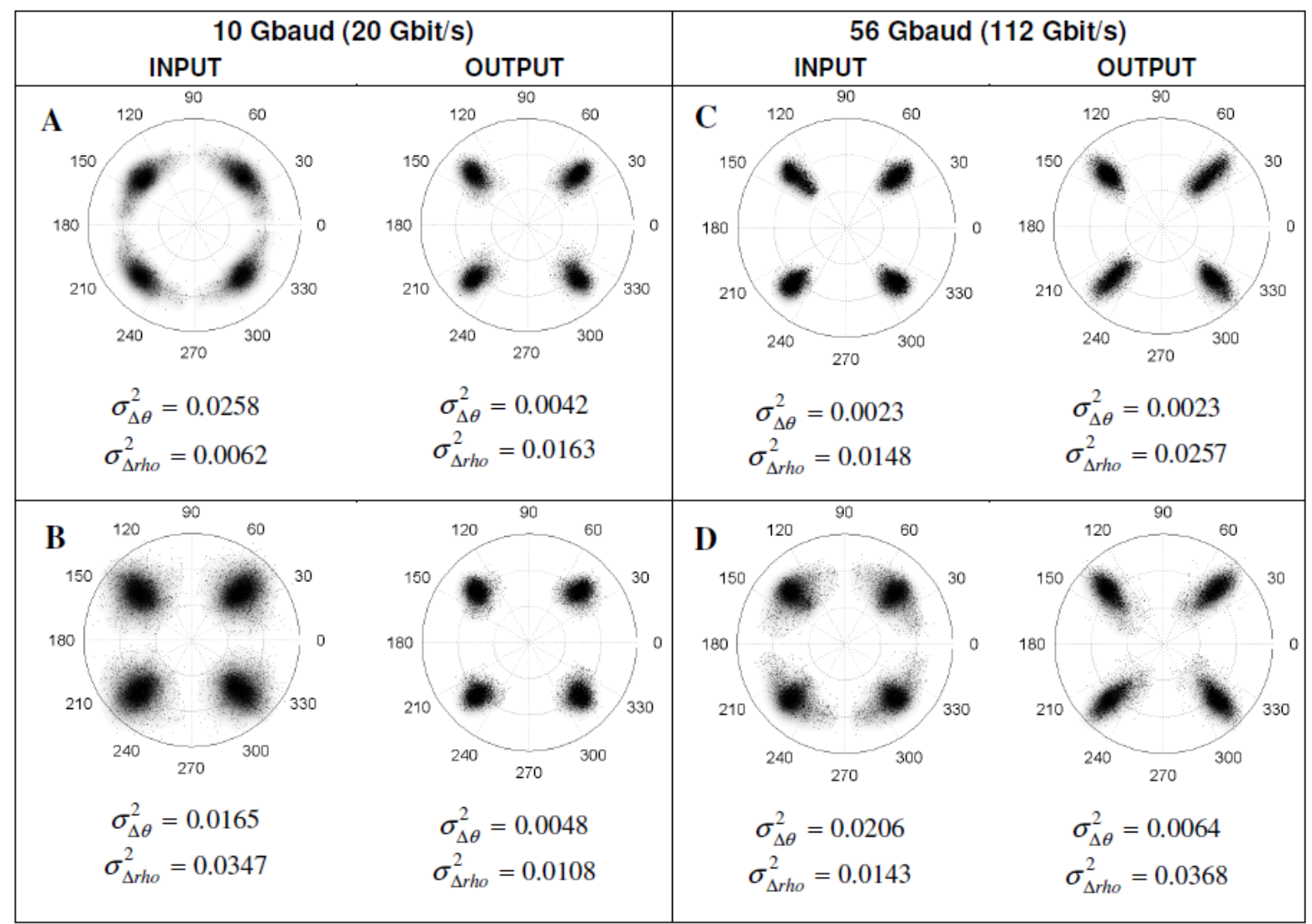

Figure 27: Regenerator performance. A - Input phase noise only; B- Phase and amplitude noise; CNo noise; D- Phase noise only. $\sigma_{\Delta \theta}^{2}$ is the phase error variance, $\sigma_{\Delta r h o}^{2}$ is the normalised amplitude noise variance. 


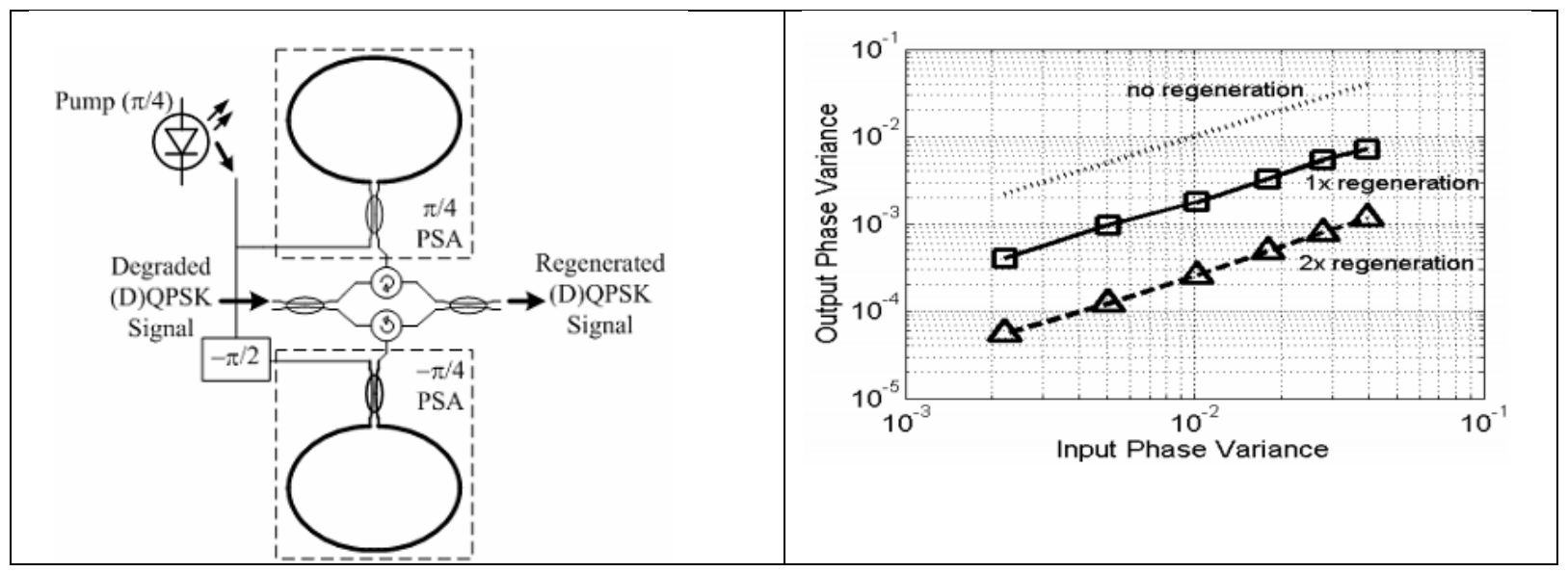

Figure 28: QPSK regenerator concept using two parallel PSAs. Left - setup. Right - Numerical simulation results [59].

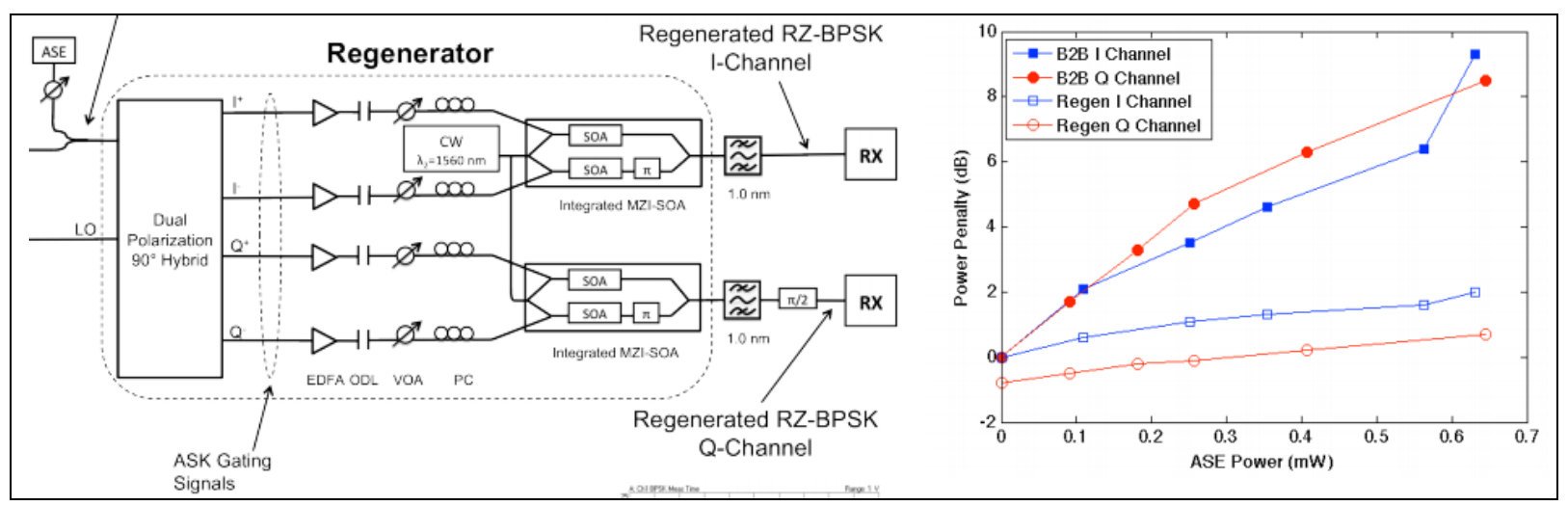

Figure 29: SOA based QPSK regenerator scheme by Kimchau et. al. Left - experimental setup. Right Power penalty measurements at BER $10^{-9}$ as ASE is added to the input signal [61].

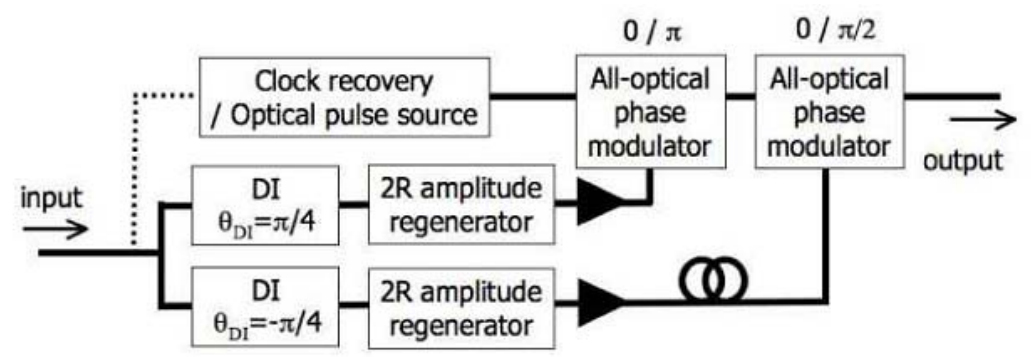

Figure 30: Block diagram of the all-optical DQPSK signal regenerator, [6]. 


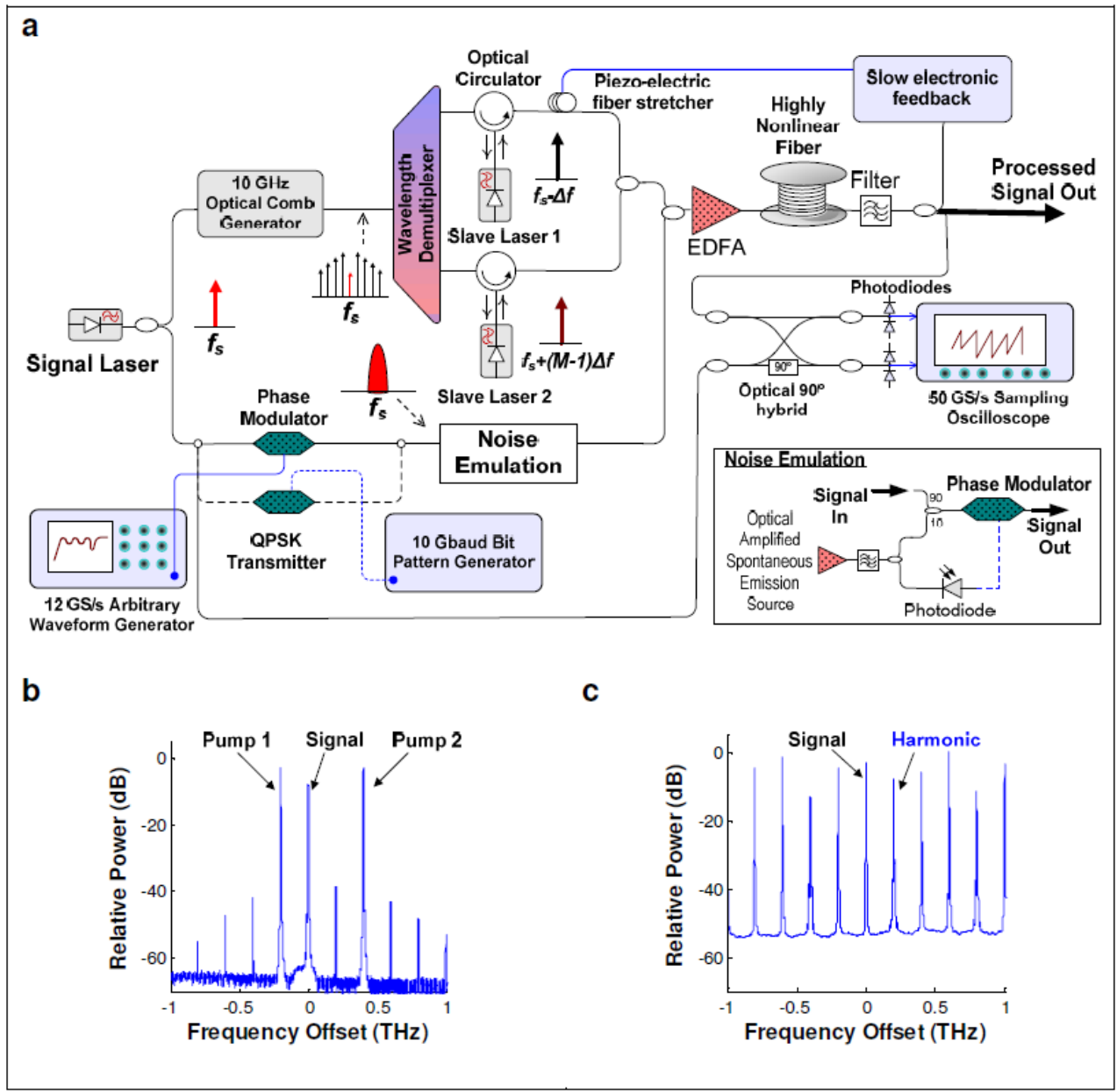

Figure 31: a, Schematic of the quantizer. EDFA, erbium doped fibre amplier. b, Optical spectrum at the input and c, output of the highly nonlinear fibre. The weak tones (over $35 \mathrm{~dB}$ less than pumps) in $b$ result from FWM in the EDFA and can be ignored [13].
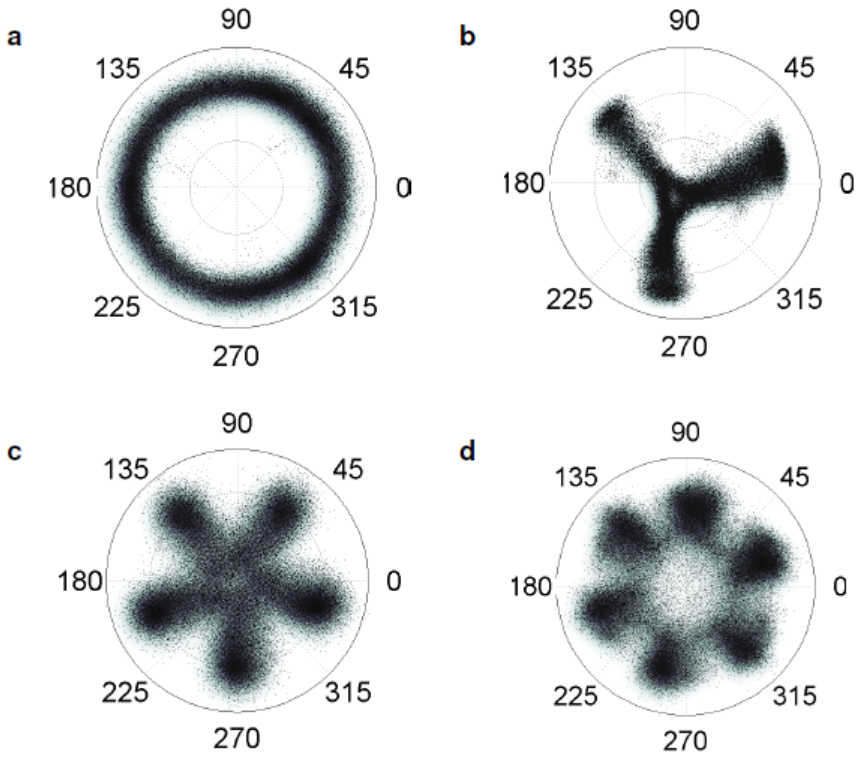

Figure 32: Signal constellation diagrams. a, Before the quantizer, signal occupies every phase state. b, After phase quantizer with pump 2 at $f_{s}+2 \Delta f$, giving 3 levels. c, After phase quantizer with pump 2 at $f_{s}+4 \Delta f$, giving 5 levels. d, After phase quantizer with pump 2 at $f_{s}+5 \Delta f$, giving 6 levels. 

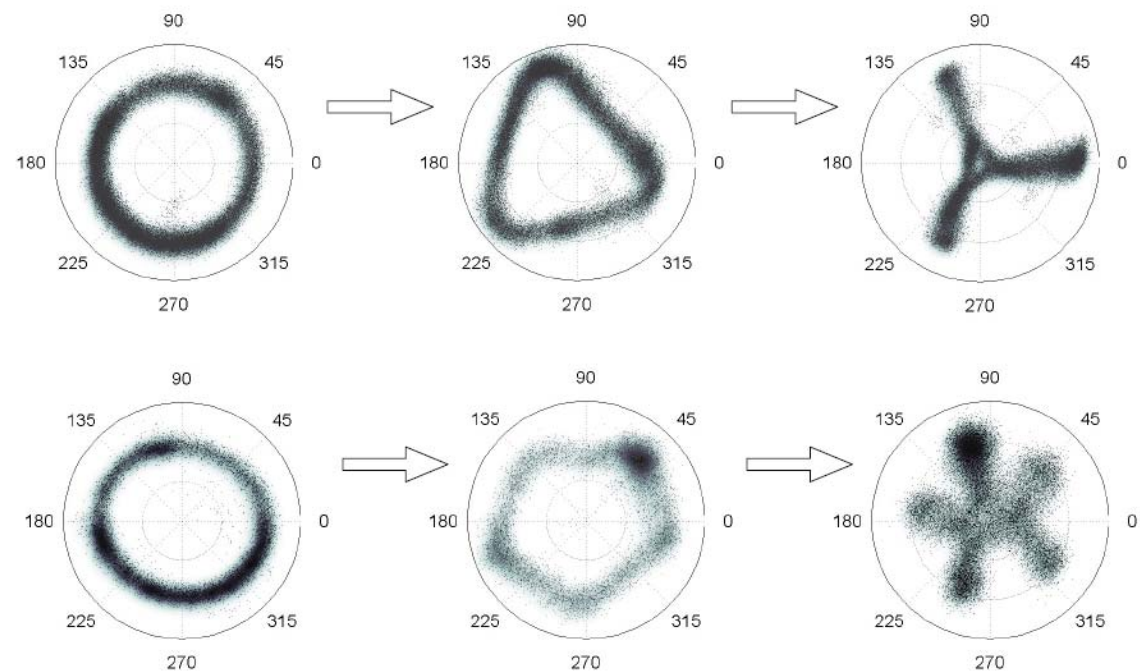

Figure 33: Output constellations with quantizer configured for 3- and 5-PSK, showing transformation as pump power is increased from zero to an optimum value.
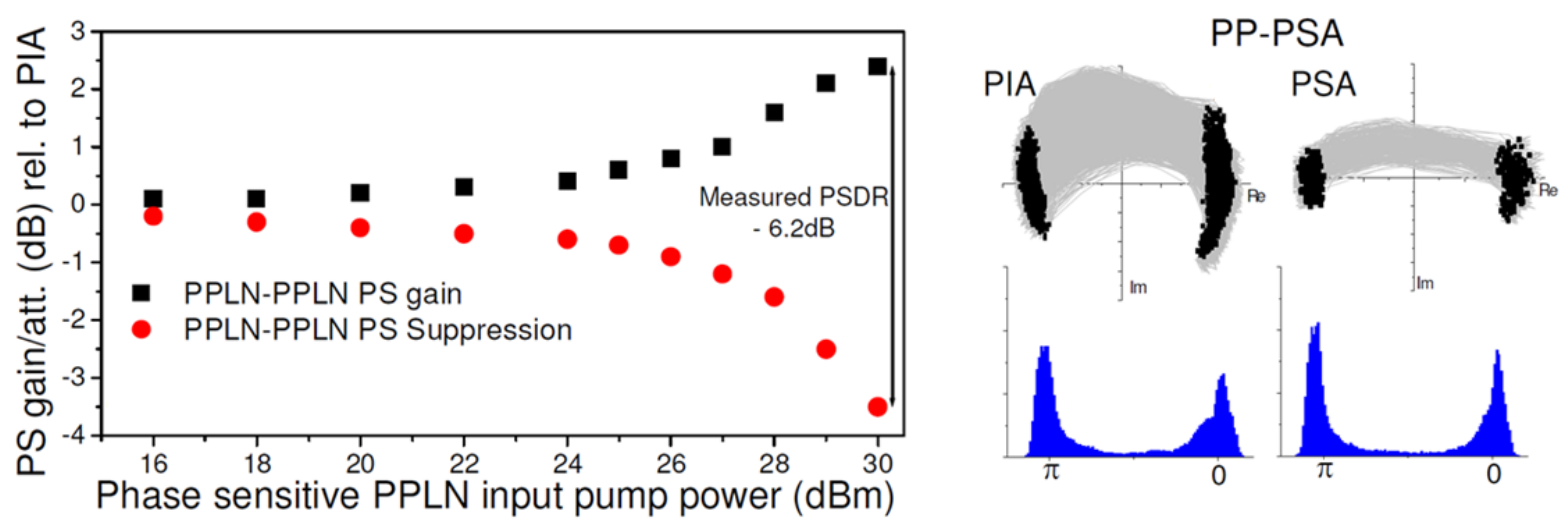

Figure 34: Characterization of a phase-squeezing PSA based on PPLN devices. Taken from [95]. 

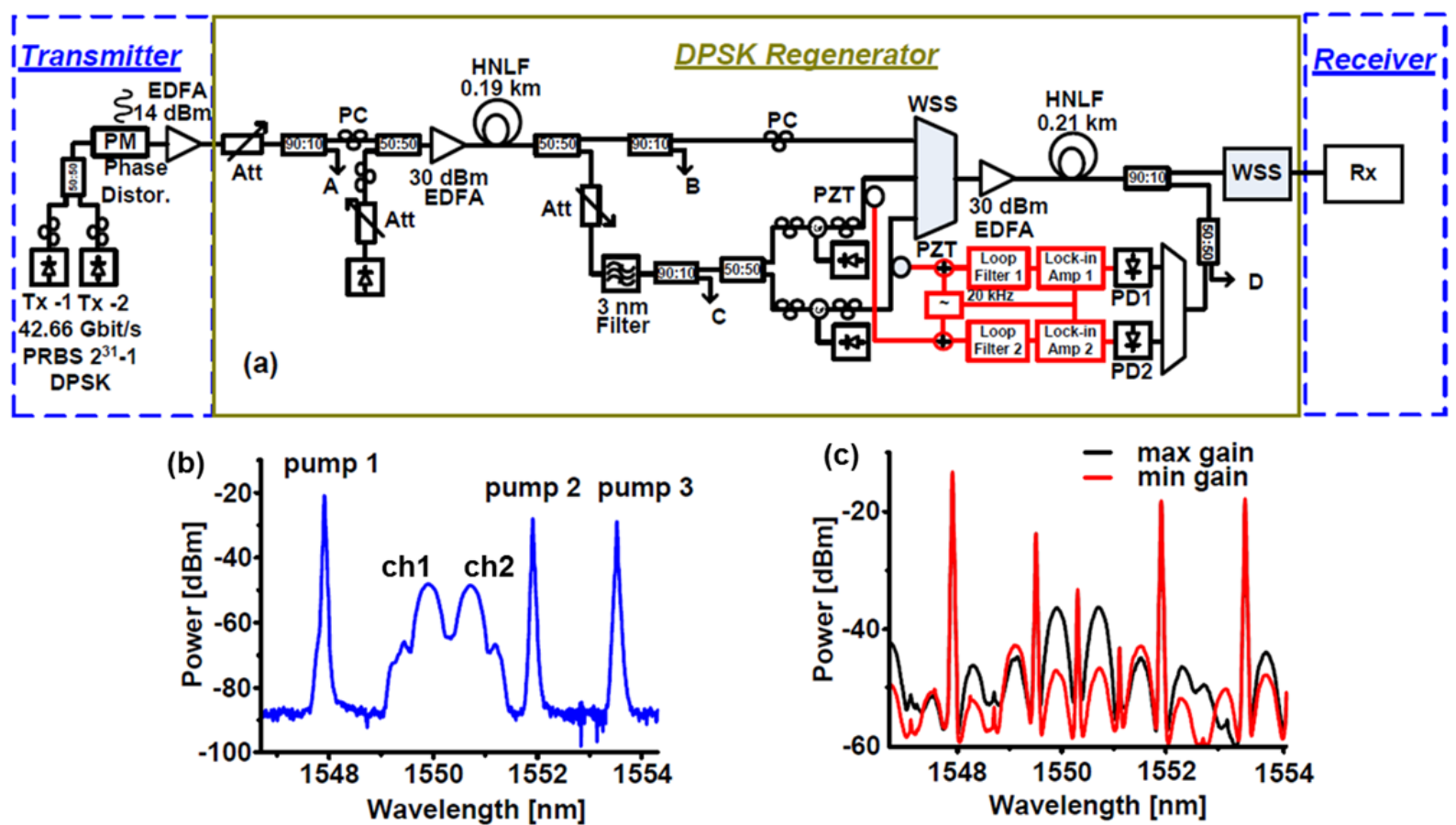

Figure 35: (a) A PS-FOPA based two-channel phase regenerator; (b) optical spectral trace of the pumps and signals at the input of the PS-FOPA, pumps 1 and 2 amplify channel 1, and pumps 1 and 3 amplify channel 2; (c) optical spectral traces at the output of the PS-FOPA. Adapted from [111].

[1] P. A. Humblet and M. Azizoglu, "On the bit error rate of lightwave systems with optical amplifiers," Journal of Lightwave Technology, vol. 9, pp. 1576-1582, 1991.

[2] M. Salsi, H. Mardoyan, P. Tran, C. Koebele, E. Dutisseuil, G. Charlet, and S. Bigo, "155x100Gbps coherent PDM-QPSK transmission over 7,200km," Proceedings of the35th European Conference on Optical Communication (ECOC), pp. 1-2, 2009.

[3] M. Salsi, C. Koebele, P. Tran, H. Mardoyan, E. Dutisseuil, J. Renaudier, M. Bigot-Astruc, L. Provost, S. Richard, P. Sillard, S. Bigo, and G. Charlet, "Transmission of 96x100Gbps with 23 percent super-FEC overhead over $11,680 \mathrm{~km}$, using optical spectral engineering," Proceedings of the 2011 Optical Fiber Communication Conference (OFC/NFOEC), pp. 1-3, 2011.

[4] S. Kumar, Impact of Nonlinearities on Fiber optic Communications, 1 ed. , 2011.

[5] M. Matsumoto, "Fiber-Based All-Optical Signal Regeneration," Selected Topics in Quantum Electronics, IEEE Journal of, vol. 18, pp. 738-752, 2012.

[6] C. McKinstrie and S. Radic, "Phase-sensitive amplification in a fiber," Optics Express, Vol. 12, Issue 20, pp. 4973-4979 (2004).

[7] K. Inoue, "Optical level equalisation based on gain saturation in fibre optical parametric amplifier," Electronics Letters, vol. 36, pp. 1016-1017, 2000.

[8] M. Matsumoto, "Regeneration of RZ-DPSK signals by fiber-based alloptical regenators," IEEE Photonics Technology Letters, vol. 17, pp. 1055-1057, 2005.

[9] M. Matsumoto, "Performance improvement of phase-shift-keying signal transmission by means of optical limiters using four-wave mixing in fibers," Journal of Lightwave Technology, vol. 23, pp. 2696-2701, 2005.

[10] C. S. Bres, A. O. J. Wiberg, J. M. Chavez-Boggio, and S. Radic, "Optical demultiplexing with extinction ratio enhancement based on 
higher order parametric interaction," Proceedings of the 35th

European Conference on Optical Communication (ECOC), pp. 1-2, 2009.

[11] A. Bogris and D. Syvridis, "RZ-DPSK signal regeneration based on dual-pump phase-sensitive amplification in fibers," IEEE Photonics Technology Letters, vol. 18, pp. 2144-2146, 2006.

[12] K. Croussore and L. Guifang, "Phase and Amplitude Regeneration of Differential Phase-Shift Keyed Signals Using Phase-Sensitive Amplification," Selected Topics in Quantum Electronics, IEEE Journal of, vol. 14, pp. 648-658, 2008.

[13] J. Kakande, R. Slavik, F. Parmigiani, A. Bogris, D. Syvridis, L. Gruner-Nielsen, R. Phelan, P. Petropoulos, and D. J. Richardson, "Multilevel quantization of optical phase in a novel coherent parametric mixer architecture," Nat Photon, vol. 5, pp. 748-752, 2011.

[14] Z. Tong, C. Lundstrom, P. A. Andrekson, C. J. McKinstrie, M. Karlsson, D. J. Blessing, E. Tipsuwannakul, B. J. Puttnam, H. Toda, and L. Gruner Nielsen, "Towards ultrasensitive optical links enabled by low-noise phase-sensitive amplifiers," Nature Photonics, vol. 5, pp. 430-436, 2011.

[15] X. Chongjin, G. Raybon, and P. J. Winzer, "Transmission of Mixed 224$\mathrm{Gb} / \mathrm{s}$ and 112-Gb/s PDM-QPSK at 50-GHz Channel Spacing Over 1200-km Dispersion-Managed LEAF Spans and Three ROADMs," Lightwave Technology, Journal of, vol. 30, pp. 547-552, 2012.

[16] S. Chandrasekhar and X. Liu, "Impact of Channel Plan and Dispersion Map on Hybrid DWDM Transmission of 42.7-Gb/s DQPSK and 10.7-Gb/s OOK on 50-GHz Grid," Photonics Technology Letters, IEEE, vol. 19, pp. $1801-1803,2007$.

[17] A. Bononi, N. Rossi, and P. Serena, "Transmission limitations due to fiber nonlinearity," Proceedings of the 2011 optical Fiber

Communication Conference and Exposition (OFC/NFOEC), and the National Fiber Optic Engineers Conference, pp. 1-3, 2011.

[18] N. J. Doran and D. Wood, "Nonlinear-optical loop mirror," optics Letters, vol. 13, pp. 56-58, 1988.

[19] K. Crecek, G. Onishchukov, K. Sponsel, A. G. Striegler, B. Schmauss, and G. Leuchs, "Experimental investigation of a modified NOLM for phase-encoded signal regeneration," Photonics Technology Letters, IEEE, vol. 18, pp. 1801-1803, 2006.

[20] C. Stephan, K. Sponsel, G. Onishchukov, B. Schmauss, and G. Leuchs, "Nonlinear Phase Noise Reduction in a DPSK Transmission System Using Cascaded Nonlinear Amplifying Loop Mirrors," Photonics Technology Letters, IEEE, vol. 21, pp. 1864-1866, 2009.

[21] M. Matsumoto, "A Fiber-Based All-Optical 3R Regenerator for DPSK Signals," IEEE Photonics Technology Letters, vol. 19, pp. 273-275, 2007.

[22] A. Fragkos, A. Bogris, D. Syvridis, and R. Phelan, "Amplitude Noise Limiting Amplifier for Phase Encoded Signals Using Injection Locking in Semiconductor Lasers," Journal of Lightwave Technology, 2011.

[23] A. Fragkos, A. Bogris, D. Syvridis, and R. Phelan, "Colorless Regenerative Amplification of Constant Envelope Phase-Modulated Optical Signals Based on Injection-Locked Fabry Perot Lasers," Photonics Technology Letters, IEEE, vol. 24, pp. 28-30, 2012.

[24] L. Quang Trung, L. Bramerie, N. Hoang Trung, M. Gay, S. Lobo, M. Joindot, J. L. Oudar, and J. C. Simon, "Saturable-Absorber-Based Phase-Preserving Amplitude Regeneration of RZ DPSK Signals,"

Photonics Technology Letters, IEEE, vol. 22, pp. 887-889, 2010.

[25] L. Quang Trung, L. Bramerie, M. Gay, M. Joindot, J. Simon, A. O'Hare, N. Hoang Trung, and J. Oudar, "All-optical phase-preserving amplitude regeneration of 28-Gbaud RZ-DQPSK signals with a microcavity saturable absorber in a recirculating loop experiment," in Optical Fiber Communication Conference and Exposition (OFC/NFOEC), 2011 and the National Fiber Optic Engineers Conference, 2011, pp. 1-3. 
[26] K. Croussore, I. Kim, C. Kim, Y. Han, and G. F. Li, "Phase-andamplitude regeneration of differential phase-shift keyed signals using a phase-sensitive amplifier," Optics Express, vol. 14, pp. 2085-2094, 2006 .

[27] D. Levandovsky, M. Vasilyev, and P. Kumar, "Amplitude squeezing of light by means of a phase-sensitive fiber parametric amplifier," optics Letters, vol. 24, pp. 984-986, 1999.

[28] H. A. Haus and J. A. Mullen, "Quantum Noise in Linear Amplifiers," Physical Review, vol. 128, p. 2407, 1962.

[29] C. M. Caves, "Quantum limits on noise in linear amplifiers," Physical Review D, vol. 26, p. 1817, 1982.

[30] R. E. Slusher and B. Yurke, "Squeezed light for coherent communications," Journal of Lightwave Technology, vol. 8, pp. 466477,1990 .

[31] J. A. Levenson, I. Abram, T. Rivera, and P. Grangier, "Reduction of quantum noise in optical parametric amplification," Journal of the Optical Society of America B, vol. 10, pp. 2233-2238, 1993.

[32] M. E. Marhic, C. H. Hsia, and J. M. Jeong, "Optical amplification in a nonlinear fibre interferometer," Electronics Letters, vol. 27, pp. 210-211, 1991.

[33] W. Imajuku, A. Takada, and Y. Yamabayashi, "Low-noise amplification under the $3 \mathrm{~dB}$ noise figure in high-gain phase-sensitive fibre amplifier," Electronics Letters, vol. 35, pp. 1954-1955, 1999.

[34] R. Tang, P. Devgan, V. S. Grigoryan, and P. Kumar, "Inline frequencynon-degenerate phase-sensitive fibre parametric amplifier for fibreoptic communication," Electronics Letters, vol. 41, pp. 1072-1074, 2005 .

[35] R. Tang, J. Lasri, P. S. Devgan, V. Grigoryan, P. Kumar, and M. Vasilyev, "Gain characteristics of a frequency nondegenerate phasesensitive fiber-optic parametric amplifier with phase self-stabilized input," Optics Express, vol. 13, pp. 10483-10493, 2005.

[36] T. Zhi, C. Lundstrom, A. Bogris, M. Karlsson, P. Andrekson, and D. Syvridis, "Measurement of sub-1dB noise figure in a non-degenerate cascaded phase-sensitive fibre parametric amplifier," Proceedings of the 35th European Conference on optical Communication (ECOC), pp. 12,2009 .

[37] C. Lundström, Z. Tong, M. Karlsson, and P. A. Andrekson, "Phase-tophase and phase-to-amplitude transfer characteristics of a nondegenerate-idler phase-sensitive amplifier," opt. Lett., vol. 36, pp. 4356-4358, 2011.

[38] W. Imajuku and A. Takada, "Gain characteristics of coherent optical amplifiers using a Mach-Zehnder interferometer with Kerr media," IEEE Journal of Quantum Electronics, vol. 35, pp. 1657-1665, 1999.

[39] M. Shirasaki and H. A. Haus, "Squeezing of pulses in a nonlinear interferometer," Journal of the optical Society of America B, vol. 7, pp. 30-34, 1990 .

[40] M. E. Marhic, Fiber optical Parametric Amplifiers, Oscillators and Related Devices, 2007.

[41] G. Agrawal, Nonlinear Fiber optics: Academic Press, 2001.

[42] J. Kakande, F. Parmigiani, R. Slavik, L. Gruner-Nielsen, D. Jakobsen, S. Herstrom, P. Petropoulos, and D. J. Richardson, "Saturation effects in degenerate phase sensitive fiber optic parametric amplifiers," in Optical Communication (ECOC), 201036 th European Conference and Exhibition on, 2010, pp. 1-3.

[43] C. Lundstrom, B. Corcoran, T. Zhi, M. Karlsson, and P. A. Andrekson, "Phase and amplitude transfer functions of a saturated phasesensitive parametric amplifier," in optical Communication (ECOC), 2011 37th European Conference and Exhibition on, 2011, pp. 1-3.

[44] M. J. Fice, A. Chiuchiarelli, E. Ciaramella, and A. J. Seeds, "Homodyne Coherent Optical Receiver Using an Optical Injection Phase- 
Lock Loop," Lightwave Technology, Journal of, vol. 29, pp. 1152-1164, 2011.

[45] A. Chiuchiarelli, M. J. Fice, E. Ciaramella, and A. J. Seeds, "Effective homodyne optical phase locking to PSK signal by means of 8b10b line coding," Optics Express, vol. 19, pp. 1707-1712, 2011.

[46] I. Kim, K. Croussore, X. Li, G. Li, T. Hasegawa, and N. Sugimoto, "All-optical carrier phase and polarization recovery using a phasesensitive oscillator," Proceedings of the 2007 Optical Fiber Communication and the National Fiber optic Engineers Conference (OFC/NFOEC), pp. 1-3, 2007.

[47] J. M. Kahn, "BPSK homodyne detection experiment using balanced optical phase-locked loop with quantized feedback," Photonics Technology Letters, IEEE, vol. 2, pp. 840-843, 1990.

[48] S. K. Ibrahim, S. Sygletos, R. Weerasuriya, and A. D. Ellis, "Novel carrier extraction scheme for phase modulated signals using feedforward based modulation stripping," in optical Communication (ECOC), 2010 36th European Conference and Exhibition on, 2010, pp. 1-3.

[49] M. J. Fice, A. J. Seeds, B. J. Pugh, J. M. Heaton, and S. J. Clements, "Homodyne coherent receiver with phase locking to orthogonal-polarisation pilot carrier by optical injection phase lock loop," in Optical Fiber Communication - incudes post deadline papers, 2009. OFC 2009. Conference on, 2009, pp. 1-3.

[50] R. Slavik, J. Kakande, and D. J. Richardson, "Feed-forward optical domain carrier recovery from high baud rate PSK signals using relatively slow electronics," in Optical Communication (ECOC), 2011

37th European Conference and Exhibition on, 2011, pp. 1-3.

[51] R. Slavik, F. Parmigiani, J. Kakande, C. Lundstrom, M. Sjodin, P. A. Andrekson, R. Weerasuriya, S. Sygletos, A. D. Ellis, L. GrunerNielsen, D. Jakobsen, S. Herstrom, R. Phelan, J. O'Gorman, A. Bogris, D. Syvridis, S. Dasgupta, P. Petropoulos, and D. J. Richardson, "Alloptical phase and amplitude regenerator for next-generation telecommunications systems," Nature Photonics, vol. 4, pp. 690-695, 2010 .

[52] R. Weerasuriya, S. Sygletos, S. K. Ibrahim, R. Phelan, J. O'Carroll, B. Kelly, J. O'Gorman, and A. D. Ellis, "Generation of frequency symmetric signals from a BPSK input for phase sensitive amplification," Proceedings of the 2010 optical Fiber Communication (OFC), pp. 1-3, 2010.

[53] J. A. Armstrong, N. Bloembergen, J. Ducuing, and P. S. Pershan, "Interactions between Light Waves in a Nonlinear Dielectric," Physical Review, vol. 127, p. 1918, 1962.

[54] R. Phelan, B. Kelly, J. O'Carroll, C. Herbert, A. Duke, and J. O'Gorman, " $-40^{\circ} \mathrm{C}<\mathrm{T}<95^{\circ} \mathrm{C}$ mode-hop-free operation of uncooled AlGaInAs-MQW discrete-mode laser diode with emission at $\lambda=1.3 \mu \mathrm{m}, "$ Electronics Letters, vol. 45, pp. 43-45, 2009.

[55] L. Gruner-Nielsen, S. Dasgupta, M. D. Mermelstein, D. Jakobsen, S. Herstrom, M. E. V. Pedersen, E. L. Lim, S. Alam, F. Parmigiani, D. Richardson, and B. Palsdottir, "A silica based highly nonlinear fibre with improved threshold for stimulated brillouin scattering," in Optical Communication (ECOC), 2010 36th European Conference and Exhibition on, 2010, pp. 1-3.

[56] L. Gruner-Nielsen, S. Herstrom, S. Dasgupta, D. Richardson, D. Jakobsen, C. Lundstrom, P. A. Andrekson, M. E. V. Pedersen, and B. Palsdottir, "Silica-based highly nonlinear fibers with a high SBS threshold," 2011 IEEE Winter Topicals (WTM), pp. 171-172, 2011.

[57] R. Slavik, A. Bogris, F. Parmigiani, J. Kakande, M. Westlund, M. Skold, L. Gruner-Nielsen, R. Phelan, D. Syvridis, P. Petropoulos, and D. J. Richardson, "Coherent All-Optical Phase and Amplitude Regenerator of Binary Phase-Encoded Signals," Selected Topics in Quantum Electronics, IEEE Journal of, vol. 18, pp. 859-869, 2012. 
[58] R. Slavik, A. Bogris, J. Kakande, F. Parmigiani, L. Gruner-Nielsen, R. Phelan, J. Vojtech, P. Petropoulos, D. Syvridis, and D. J. Richardson, "Field-Trial of an All-Optical PSK

Regenerator/Multicaster in a 40 Gbit/s, 38 Channel DWDM Transmission Experiment," Lightwave Technology, Journal of, vol. 30, pp. 512-520, 2012 .

[59] Z. Zheng, L. An, Z. Li, X. Zhao, and X. Liu, "All-optical regeneration of DQPSK/QPSK signals based on phase-sensitive amplification," Optics Communications, vol. 281, pp. 2755-2759, 2008.

[60] M. Matsumoto, "All-optical DQPSK signal regeneration using $2 \mathrm{R}$ amplitude regenerators," Optics Express, vol. 18, pp. 10-24, 2010.

[61] N. N. Kimchau, K. Tomofumi, M. G. John, P. Henrik, and J. B. Daniel, "All-optical 2R Regeneration of BPSK and QPSK Data using a 90 degree Optical Hybrid and Integrated SOA-MZI Wavelength Converter Pairs," Proceedings of the 2011 optical Fiber Communication Conference (OFC/NFOEC), p. OMT3, 2011.

[62] G. C. Valley, "Photonic analog-to-digital converters," optics Express, vol. 15, pp. 1955-1982, 2007.

[63] R. H. Walden, "Analog-to-digital converter survey and analysis," IEEE Journal on Selected Areas in Communications, vol. 17, pp. 539-550, 1999.

[64] J. Kakande, A. Bogris, R. Slavik, F. Parmigiani, D. Syvridis, P. Petropoulos, and D. J. Richardson, "First demonstration of alloptical QPSK signal regeneration in a novel multi-format phase sensitive amplifier," Proceedings of the 36th European Conference and Exhibition on Optical Communication (ECOC), pp. 1-3, 2010.

[65] J. Kakande, A. Bogris, R. Slavik, F. Parmigiani, D. Syvridis, P. Petropoulos, D. Richardson, M. Westlund, and M. Skold, "QPSK phase and amplitude regeneration at 56 Gbaud in a novel idler-free nondegenerate phase sensitive amplifier," Proceedings of the 2011 optical Fiber Communication Conference (OFC/NFOEC), p. OMT4, 2011.

[66] C. Yu, S. Zhang, P. Y. Kam, and J. Chen, "Bit-Error Rate Performance of Coherent Optical M-ary PSK/QAM using Decision-Aided Maximum Likelihood Phase Estimation," Optics Express, vol. 18, pp. 1208812103,2010 .

[67] F. Da Ros and C. Peucheret, "QPSK phase regeneration in saturated degenerate dual-pump phase sensitive amplifiers," in Photonics Conference (PHO), 2011 IEEE, 2011, pp. 105-106.

[68] M. Hirano, T. Nakanishi, T. Okuno, and M. Onishi, "Silica-Based Highly Nonlinear Fibers and Their Application," Selected Topics in Quantum Electronics, IEEE Journal of, vol. 15, pp. 103-113, 2009.

[69] E. Myslivets, C. Lundstrom, J. M. Aparicio, S. Moro, A. O. J. Wiberg, C. S. Bres, N. Alic, P. A. Andrekson, and S. Radic, "Spatial Equalization of Zero-Dispersion Wavelength Profiles in Nonlinear Fibers," IEEE Photonics Technology Letters, vol. 21, pp. 1807-1809, 2009.

[70] L. Ju Han, T. Nagashima, T. Hasegawa, S. Ohara, N. Sugimoto, and K. Kikuchi, "Bismuth-oxide-based nonlinear fiber with a high SBS threshold and its application to four-wave-mixing wavelength conversion using a pure continuous-wave pump," Lightwave Technology, Journal of, vol. 24, pp. 22-28, 2006.

[71] F. Parmigiani, S. Asimakis, N. Sugimoto, F. Koizumi, P. Petropoulos, and D. J. Richardson, "2R regenerator based on a 2-m-long highly nonlinear bismuth oxide fiber," Opt. Express, vol. 14, pp. 5038-5044, 2006 .

[72] F. Poletti, X. Feng, G. M. Ponzo, M. N. Petrovich, W. H. Loh, and D. J. Richardson, "All-solid highly nonlinear singlemode fibers with a tailored dispersion profile," Opt. Express, vol. 19, pp. 66-80, 2011.

[73] J. S. Sanghera, L. Brandon Shaw, and I. D. Aggarwal, "Chalcogenide Glass-Fiber-Based Mid-IR Sources and Applications," Selected Topics in Quantum Electronics, IEEE Journal of, vol. 15, pp. 114-119, 2009. 
[74] J.Y.Y. Leong, P. Petropoulos, J. H. V. Price, H. EbendorffHeidepriem, S. Asimakis, R. C. Moore, K. E. Frampton, V. Finazzi, X. Feng, T. M. Monro, and D. J. Richardson, "High-nonlinearity dispersion-shifted lead-silicate holey fibers for efficient 1-um pumped supercontinuum generation," Lightwave Technology, Journal of, vol. 24, pp. 183-190, 2006.

[75] S. Afshar V, W. Q. Zhang, H. Ebendorff-Heidepriem, and T. M. Monro, "Small core optical waveguides are more nonlinear than expected: experimental confirmation," opt. Lett., vol. 34, pp. 3577-3579, 2009.

[76] S. D. Le, D. M. Nguyen, M. Thual, L. Bramerie, M. Costa e Silva, K. Lenglé, M. Gay, T. Chartier, L. Brilland, D. Méchin, P. Toupin, and J. Troles, "Efficient four-wave mixing in an ultra-highly nonlinear suspended-core chalcogenide As38Se62 fiber," opt. Express, vol. 19, pp. B653-B660, 2011.

[77] D.-I. Yeom, E. C. Mägi, M. R. E. Lamont, M. A. F. Roelens, L. Fu, and B. J. Eggleton, "Low-threshold supercontinuum generation in highly nonlinear chalcogenide nanowires," opt. Lett., vol. 33, pp. 660-662, 2008 .

[78] B. J. Eggleton, B. Luther-Davies, and K. Richardson, "Chalcogenide photonics," Nat Photon, vol. 5, pp. 141-148, 2011.

[79] C. Fortier, J. Fatome, S. Pitois, F. Smektala, G. Millot, J. Troles, F. Desevedavy, P. Houizot, L. Brilland, and N. Traynor, "Experimental investigation of Brillouin and Raman scattering in a 2SG sulfide glass microstructured chalcogenide fiber," opt. Express, vol. 16, pp. 9398-9404, 2008 .

[80] L. Fu, M. Rochette, V. Ta'eed, D. Moss, and B. Eggleton, "Investigation of self-phase modulation based optical regeneration in single mode As2se3 chalcogenide glass fiber," opt. Express, vol. 13, pp. 7637-7644, 2005.

[81] M. A. Ettabib, L. Jones, J. Kakande, R. Slavik, F. Parmigiani, X. Feng, F. Poletti, G. M. Ponzo, J. A. Shi, M. N. Petrovich, W. H. Loh, P. Petropoulos, and D. J. Richardson, "Phase sensitive amplification in a highly nonlinear lead-silicate fiber," opt. Express, vol. 20, pp. 1629-34, 2012 .

[82] M. Asobe, T. Kanamori, and K. Kubodera, "Applications of highly nonlinear chalcogenide glass fibers in ultrafast all-optical switches," Quantum Electronics, IEEE Journal of, vol. 29, pp. 23252333, 1993 .

[83] K. Suzuki, Y. Hamachi, and T. Baba, "Fabrication and characterization of chalcogenide glass photonic crystal waveguides," opt. Express, vol. 17, pp. 22393-22400, 2009.

[84] M. D. Pelusi, V. G. Ta'eed, F. Libin, E. Magi, M. R. E. Lamont, S. Madden, C. Duk-Yong, D. A. P. Bulla, B. Luther-Davies, and B. J. Eggleton, "Applications of Highly-Nonlinear Chalcogenide Glass Devices Tailored for High-Speed All-Optical Signal Processing," Selected Topics in Quantum Electronics, IEEE Journal of, vol. 14, pp. 529-539, 2008 .

[85] M. R. Lamont, B. Luther-Davies, D.-Y. Choi, S. Madden, and B. J. Eggleton, "Supercontinuum generation in dispersion engineered highly nonlinear $(\gamma=10 / \mathrm{W} / \mathrm{m})$ As2S3 chalcogenide planar waveguide," opt. Express, vol. 16, pp. 14938-14944, 2008.

[86] X. Gai, S. Madden, D.-Y. Choi, D. Bulla, and B. Luther-Davies, "Dispersion engineered Ge11.5As24Se64.5 nanowires with a nonlinear parameter of $136 \mathrm{~W}-1 \mathrm{~m}-1$ at $1550 \mathrm{~nm}, "$ opt. Express, vol. 18, pp. 18866$18874,2010$.

[87] T. Vallaitis, S. Bogatscher, L. Alloatti, P. Dumon, R. Baets, M. L. Scimeca, I. Biaggio, F. Diederich, C. Koos, W. Freude, and J. Leuthold, "Optical properties of highly nonlinear silicon-organic hybrid (SOH) waveguide geometries," opt. Express, vol. 17, pp. 1735717368, 2009. 
[88] J. Leuthold, C. Koos, and W. Freude, "Nonlinear silicon photonics," Nat Photon, vol. 4, pp. 535-544, 2010.

[89] C. Koos, P. Vorreau, T. Vallaitis, P. Dumon, W. Bogaerts, R. Baets, B. Esembeson, BiaggioI, T. Michinobu, F. Diederich, W. Freude, and J. Leuthold, "All-optical high-speed signal processing with siliconorganic hybrid slot waveguides," Nature Photonics, vol. 3, pp. 216219,2009 .

[90] M. A. Foster, A. C. Turner, J. E. Sharping, B. S. Schmidt, M. Lipson, and A. L. Gaeta, "Broad-band optical parametric gain on a silicon photonic chip," Nature, vol. 441, pp. 960-963, 2006.

[91] R. Salem, M. A. Foster, A. C. Turner, D. F. Geraghty, M. Lipson, and A. L. Gaeta, "Signal regeneration using low-power four-wave mixing on silicon chip," Nat Photon, vol. 2, pp. 35-38, 2008.

[92] L. K. Oxenlowe, J. Hua, M. Galili, P. Minhao, H. Hao, H. C. H. Mulvad, K. Yvind, J. M. Hvam, A. T. Clausen, and P. Jeppesen, "Silicon Photonics for Signal Processing of Tbit/s Serial Data Signals," Selected Topics in Quantum Electronics, IEEE Journal of, vol. 18, pp. 996-1005, 2012.

[93] K. J. Lee, F. Parmigiani, S. Liu, J. Kakande, P. Petropoulos, K. Gallo, and D. Richardson, "Phase sensitive amplification based on quadratic cascading in a periodically poled lithium niobate waveguide," Optics Express, vol. 17, pp. 20393-20400, 2009.

[94] S. Liu, K. J. Lee, F. Parmigiani, J. Kakande, K. Gallo, P. Petropoulos, and D. J. Richardson, "Phase-regenerative wavelength conversion in periodically poled lithium niobate waveguides," opt. Express, vol. 19, pp. 11705-11715, 2011.

[95] B. J. Puttnam, D. Mazroa, S. Shinada, and N. Wada, "Phase-squeezing properties of non-degenerate PSAs using PPLN waveguides," Opt.

Express, vol. 19, pp. B131-B139, 2011.

[96] F. Gomez-Agis, C. M. Okonkwo, A. Albores-Mejia, E. Tangdiongga, and H. J. S. Dorren, "320-to-10 Gbit/s all-optical demultiplexing using sum-frequency generation in PPLN waveguide," Electronics Letters, vol. 46, pp. 1008-1009, 2010.

[97] A. Bogoni, W. Xiaoxia, S. R. Nuccio, and A. E. Willner, "640 Gb/s All-Optical Regenerator Based on a Periodically Poled Lithium Niobate Waveguide," Lightwave Technology, Journal of, vol. 30, pp. 1829-1834, 2012 .

[98] H. Hao, R. Nouroozi, R. Ludwig, C. Schmidt-Langhorst, H. Suche, W. Sohler, and C. Schubert, "Polarization-Insensitive 320-Gb/s In-Line All-Optical Wavelength Conversion in a 320-km Transmission Span," Photonics Technology Letters, IEEE, vol. 23, pp. 627-629, 2011.

[99] G.-W. Lu and T. Miyazaki, "Optical phase erasure based on FWM in HNLF enabling format conversion from 320-Gb/s RZDQPSK to 160-Gb/s RZDPSK," Optics Express, vol. 17, pp. 13346-13353, 2009.

[100] A. E. Willner, O. F. Yilmaz, W. Jian, W. Xiaoxia, A. Bogoni, Z. Lin, and S. R. Nuccio, "Optically Efficient Nonlinear Signal Processing," Selected Topics in Quantum Electronics, IEEE Journal of, vol. 17, pp. $320-332,2011$.

[101] A. Poustie, "SOA-based All-optical Processing," in optical Fiber Communication and the National Fiber optic Engineers Conference, 2007. OFC/NFOEC 2007. Conference on, 2007, pp. 1-38.

[102] T. Akiyama, M. Sugawara, and Y. Arakawa, "Quantum-Dot Semiconductor Optical Amplifiers," Proceedings of the IEEE, vol. 95, pp. 1757-1766, 2007 .

[103] K. Hinton, G. Raskutti, P. M. Farrell, and R. S. Tucker, "Switching Energy and Device Size Limits on Digital Photonic Signal Processing Technologies," Selected Topics in Quantum Electronics, IEEE Journal of, vol. 14, pp. 938-945, 2008.

[104] O. Wada, "Recent Progress in Semiconductor-Based Photonic SignalProcessing Devices," Selected Topics in Quantum Electronics, IEEE Journal of, vol. 17, pp. 309-319, 2011. 
[105] E. Tangdiongga, Y. Liu, H. de Waardt, G. D. Khoe, A. M. J. Koonen, H. J. S. Dorren, X. Shu, and I. Bennion, "All-optical demultiplexing of 640 to 40 Gbits/s using filtered chirp of a semiconductor optical amplifier," opt. Lett., vol. 32, pp. 835-837, 2007.

[106] S. Nakamura, Y. Ueno, and K. Tajima, "Error-free all-optical demultiplexing at $336 \mathrm{~Gb} / \mathrm{s}$ with a hybrid-integrated symmetric-MachZehnder switch," in Optical Fiber Communication Conference and Exhibit, 2002. OFC 2002, 2002, pp. FD3-1-FD3-3.

[107] T. Hirooka, M. Okazaki, T. Hirano, P. Guan, M. Nakazawa, and S. Nakamura, "All-optical Demultiplexing of 640-Gb/s OTDM-DPSK Signal Using a Semiconductor SMZ Switch," Photonics Technology Letters, IEEE, vol. 21, pp. 1574-1576, 2009.

[108] M. Bougioukos, C. Kouloumentas, M. Spyropoulou, G. Giannoulis, D. Kalavrouziotis, A. Maziotis, P. Bakopoulos, R. Harmon, D. Rogers, J. Harrison, A. Poustie, G. Maxwell, and H. Avramopoulos, "Multi-format all-optical processing based on a large-scale, hybridly integrated photonic circuit," Optics Express, vol. 19, pp. 11479-11489, Jun 6 2011 .

[109] R. P. Webb, J. M. Dailey, R. J. Manning, and A. D. Ellis, "Phase discrimination and simultaneous frequency conversion of the orthogonal components of an optical signal by four-wave mixing in an SOA," Opt. Express, vol. 19, pp. 20015-20022, 2011.

[110] B. J. Puttnam, A. Szabo, D. Mazroa, S. Shinada, and N. Wada, "Multichannel phase squeezing in a PPLN-PPLN PSA," in optical Fiber Communication Conference and Exposition (OFC/NFOEC), 2012 and the National Fiber optic Engineers Conference, 2012, pp. 1-3.

[111] S. Sygletos, P. Frascella, S. K. Ibrahim, L. Grüner-Nielsen, R. Phelan, J. O'Gorman, and A. D. Ellis, "A practical phase sensitive amplification scheme for two channel phase regeneration," Opt.

Express, vol. 19, pp. B938-B945, 2011.

[112] K. Cvecek, K. Sponsel, C. Stephan, G. Onishchukov, R. Ludwig, C. Schubert, B. Schmauss, and G. Leuchs, "Phase-preserving amplitude regeneration for a WDM RZ-DPSK signal using a nonlinear amplifying loop mirror," opt. Express, vol. 16, pp. 1923-1928, 2008.

[113] C. Kouloumentas, P. Vorreau, L. Provost, P. Petropoulos, W. Freude, J. Leuthold, and I. Tomkos, "All-Fiberized Dispersion-Managed Multichannel Regeneration at $43 \mathrm{~Gb} / \mathrm{s}, "$ IEEE Photonics Technology Letters, vol. 20, pp. 1854-6, 2008.

[114] F. Parmigiani, L. Provost, P. Petropoulos, D. J. Richardson, W. Freude, J. Leuthold, A. D. Ellis, and I. Tomkos, "Progress in Multichannel All-Optical Regeneration Based on Fiber Technology," Selected Topics in Quantum Electronics, IEEE Journal of, vol. 18, pp. $689-700,2012$.

[115] M. Hierold, T. Roethlingshoefer, K. Sponsel, G. Onishchukov, B. Schmauss, and G. Leuchs, "Multilevel Phase-Preserving Amplitude Regeneration Using a Single Nonlinear Amplifying Loop Mirror," IEEE Photonics Technology Letters, vol. 23, pp. 1007-1009, 2011.

[116] P. T. Rakich, C. Reinke, R. Camacho, P. Davids and Z. Wang, Phys. Rev. X 2, 011008 (2012). 